\title{
RFID Grids: Part II—Experimentations
}

\author{
Stefano Caizzone and Gaetano Marrocco
}

\begin{abstract}
The RFID Grid is a model for generally coupled multitudes of tags including single-chip tags in close mutual proximity or a single tag with a plurality of embedded microchips. Some properties of this new entity, useful for passive Sensing and for Security, are the possibility to increase the read-range and to provide responses rather insensitive to the interrogation modalities. These recently introduced issues are here experimented for the first time with many real-world examples comprising multi-chip configurations designed for improved power scavenging and for passive sensing of things.
\end{abstract}

Index Terms-Array, grid, MIMO, remote sensing, RFID, sensor, space diversity.

\section{INTRODUCTION}

V ERY recently, the paper [1] introduced the concept of RFID Grid, e.g., a generally coupled multitude of UHF (or higher frequency) RFID tags, including single-chip tags in close mutual proximity or a single tag with a plurality of embedded microchips. This model is useful to predict the impedance and the backscattering performances of tags over a dense collection of objects such as items over a shelf, as well as to design new sensing-oriented tags. RFID tags, indeed, may be equipped with more than a single chip to communicate the identity of the object, by one of the chip, and its physical state by means of the other chip or a combination of both [2]-[4].

In [1] the basic equations for the direct and inverse links were derived by using the very general model of multi-port loaded scatterers. The theoretical analysis has permitted to discover new features, some of them common to MIMO and Space-Diversity systems [5], and in particular the possibility

i) to handle the inter-antenna coupling in a useful way with the purpose to improve the power harvested by the microchip and hence to enlarge the read range;

ii) to achieve measured analog backscattered information which are rather independent on the mutual position between reader and tags themselves (distance and orientation). Such a function, denoted as Analog Identifier (A-Id) as complementation of the Digital Identifier (D-Id) normally stored in the microchip memory, originates from the combined processing of direct- and

Manuscript received October 16, 2010; revised December 14, 2010; accepted January 24, 2011. Date of publication June 09, 2011; date of current version August 03, 2011.

S. Caizzone was with the DISP-University of Roma Tor Vergata Via del Politecnico, 1, 00133 Roma, Italy . He is now with the Antenna Group, Institute of Communications and Navigation, German Space Agency (DLR), 82230 Wessling, Germany.

G. Marrocco is with the DISP-University of Roma Tor Vergata Via del Politecnico, 1, 00133 Roma, Italy (e-mail: marrocco@disp.uniroma2.it).

Color versions of one or more of the figures in this paper are available online at http://ieeexplore.ieee.org.

Digital Object Identifier 10.1109/TAP.2011.2158974 inverse-link measurements. A-Id could have significant application in the context of sensing and security. In both cases, indeed, the analog response of the tag (basically the level of the backscattered power or the turn-on power) is processed in addition to the digital microchip identifier, to earn some physical information about the tag's identity (security) and about the variation of the tagged object of the nearby environment (sensing). At these purposes it is of primary importance that the reading of analog information from the tag is as more independent as possible on the interrogation modality, in order to easily collect the tag's response at different times without the need to strictly replicate a specific reader-tag set-up. In particular, the availability of reading-robust functions could boost the emerging pervasive sensing and context aware applications within the Internet of Things paradigm [6].

The wireless characterization of RFID tags has been mainly focused to the measurement of the tag's read range or radiation patterns starting from the experimental evaluation of the threshold (or turn-on) power level [7] of the tag. Another performance parameter, commonly measured, is the backscattered power from the tag to the reader's detector or, more in general, the tag's radar cross section [8], [9]. Both turn-on power and RCS are related to gain and input impedance of the tag and therefore they cannot be easily de-embedded by means of conventional wireless measurements techniques. The characterization of tag input impedance is instead generally performed by using balanced probes and de-embedding procedures as in [10].

The response of RFID grids has never been characterized in a systematic way: some studies [11]-[16] have been conducted in order to assess the overall influence of mutual coupling between tags over the degradation of the RFID link and the results are mainly aiming at mitigating this degradation (see [1] for an extended discussion).

On the other hand, in the perspective of understanding the coupling between tags to exploit useful features, this paper presents a detailed experimental campaign to prove and discuss some of the concepts introduced in the previous companion paper [1] by the help of real-world examples having incremental complexity. Starting from a tag with a single microchip, the experiments go on through integrated two-microchips tags, up to $3 \times 3$ RFID grids. Measurements have been performed in presence of different reader-grid positions, time-varying tagged objects and non-stationary environments. The aim is to demonstrate that the RFID-oriented analytical formulas provided in [1] can be useful to dominate the inter-port coupling within the design task and to verify the true degree of invariance of the analog identifier in uncontrolled conditions.

The paper is organized as follows. Section II introduces the measurement procedures for the relevant RFID responses, 
including angle- and distance-invariant analog identifiers, the turn-on power and the realized gain. A single-chip tag in standalone configuration is measured in Section III to understand the quality of responses achievable in absence of inter-tag interactions and of other ambient perturbation. Three examples of two-chips grids are then considered in detail in Section IV, with particular attention to evaluate the improvement in the RFID performances by a systematic design of the grid, and to characterize the angular invariance of the analog identifier also in sensing-like applications. Finally, a $3 \times 3$ grid of commercial tags is explored in Section V where the sensitivity of the fingerprint is experienced with respect to the presence of metallic scatterers close to the interrogating reader.

\section{BACKGROUND AND MEASUREMENT METHODOLOGY}

According to [1], the power scavenging performances of an RFID Grid are completely characterized in the free space by means of the embedded realized gain of each $n$th port

$$
\tilde{G}_{n}=4 \eta_{0} R_{C, n}\left|\left[\mathbf{Y}_{G}\right]_{n} \cdot \mathbf{g}\right|^{2}
$$

where $\left[\mathbf{Y}_{G}\right]_{n}$ is the $n$th row of the admittance matrix $\mathbf{Y}_{\mathbf{G}}=$ $\left(\mathbf{Z}_{C}+\mathbf{Z}\right)^{-1}$ of the grid, being $\mathbf{Z}$ the impedance matrix referred to the RFID microchips' connections, and $\mathbf{Z}_{C}$ a diagonal matrix containing the equivalent impedances $\left\{Z_{C, n}=R_{C, n}+\right.$ $\left.j X_{C, n}\right\}$ of the microchips. The vector $\mathrm{g}$ of elements $g_{n}=$ $\sqrt{R_{n n} G_{n}(\underline{\hat{r}}) \chi_{n} / \eta_{0}} e^{j \Phi_{n}(\underline{\hat{r}})}$ embeds all the electromagnetic parameters associated with the ports, e.g., the open circuit gain $G_{n}$, the polarization mismatch $\chi_{n}$, the self resistance $R_{n n}$ and finally the phase $\Phi_{n}$ of the embedded field. The gain $\tilde{G}_{n}$ is the parameter to be maximized (in the useful direction) for best power scavenging and hence for longest read distance.

Physical information about the Grid and about its interaction with the tagged objects can be extracted from the analog identifier or fingerprint $F_{n}$ of the $n$th port, independently on the reading modalities. The analog identifier has been defined in [1] as a function of the turn-on power $P_{n}^{t o}$ of the $n$th port and of the corresponding backscattered power $P_{R \leftarrow T n}$ collected by the reader. For the particular modulation scheme for which the microchip' impedance is switched between a high impedance and the scavenging impedance $Z_{C, n}$, the analog identifier may be written in a very compact form

$$
F_{n} \equiv \frac{p_{n}}{\sqrt{P_{R \leftarrow T n} P_{n}^{t o}}} \propto R_{C, n}\left|Y_{G, n n}\right|
$$

where $p_{n}$ is the sensitivity of the microchip at the $n$th port, and the proportionality factor accounts for the reader's front-end and the modulation scheme, as discussed later on.

The particular case of the single-chip tag yields

$$
F=\propto \frac{R_{C}}{\left|Z_{A}+Z_{C}\right|}
$$

where $Z_{A}$ is simply the input impedance of the antenna of the tag. Both the above expressions may be evaluated along with the frequency to carry on further informations.
The physical meaning of analog identifier, and its calculation from experimental data deserves some clarification. It combines, by definition, measured data from both the direct (power harvesting) and reverse (power backscattering) links. Under the hypothesis of a stationary environment between the reader's interrogation and the tag's responses, any interaction with the nearby environment, and the mutual distance and orientation between the reader and the RFID systems, will affect both the links in a same manner, thanks to the antenna reciprocity. Hence, it was demonstrated that the simple combination of turn-on power and backscattered power will mathematically remove any kind of the above mentioned electromagnetic interactions. What remains is the functional dependence on the $n$th diagonal entry $Y_{G, n n}$ of the complete admittance matrix, in the case of multichip systems, or the dependence on the series connection between the input impedance of the tag's antenna and of the microchip in the simpler case of one-chip tag. In this sense, the A-Id is a structural property of the (multi-port) tag. Since the network admittance matrix is sensitive to the object where the tag is attached on, the variation of the A-Id in successive measurements may be related to time-changing chemical-physical properties of the object, therefore giving sensing capability. The orientation-invariance of A-Id will permit to perform this measurement without taking care to replicate a same reader-tag position at successive times.

The calculation of A-Id requires the knowledge of the turn-on power $P_{n}^{t o}$. At this purpose the power entering into the reader's antenna is gradually increased, in a controlled way, till the $n$th chip begins responding. At that moment, such a value of input power is the required turn-on $P_{i n}^{t o}$ and the corresponding backscattered power $P_{R \leftarrow T n}$ received by the reader is stored on a Personal Computer. In case of multi-chips configurations, an inventory task is preliminary performed, e.g., all the chips' identifiers are collected, and then each chip is interrogated sequentially.

Usually, commercial readers return backscattered power in the form of RSSI (received signal strength indication), after a process of demodulation, frequency conversion and equalization. The particular relationship between $P_{R \leftarrow T n}$ and the RSSI is hardware-dependent but often takes the form

$$
P_{R \leftarrow T n}(d B)=K_{1} R S S I+K_{2}
$$

with $K_{1,2}$ are specific to the reader's chipset but also to the modulation scheme. In all the measurements shown in this work, the stored RSSI signal is related to the high-level modulation state. Anyway, the experimentally-evaluated A-Id from (2) and (4) is not an absolute fingerprint of the tag but it is filtered by the reader's circuitry. The use of A-Id for sensing will therefore requires a same reader family for subsequent interrogations to avoid other kinds of uncertainties.

Finally, the A-Id depends on the microchips' impedance that, as its is well known, exhibits a non-linear dependence on the impinging interrogation power. The proposed procedure to derive the A-Id, however, enforces the turn-on power condition: it means that the microchip will always receive the same amount of power, just corresponding to its sensitivity. Thanks to this 


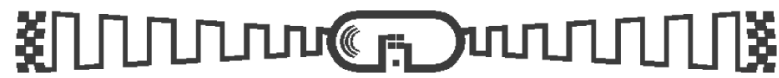

Fig. 1. The LabID UHF tag used in the experimentation campaign.

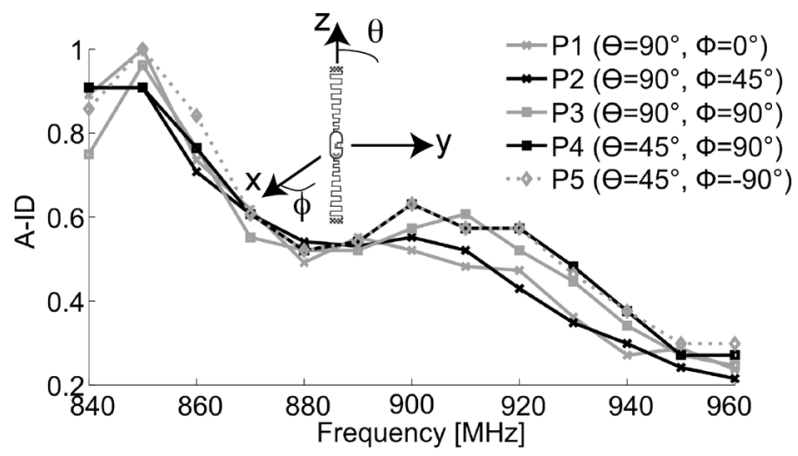

a)

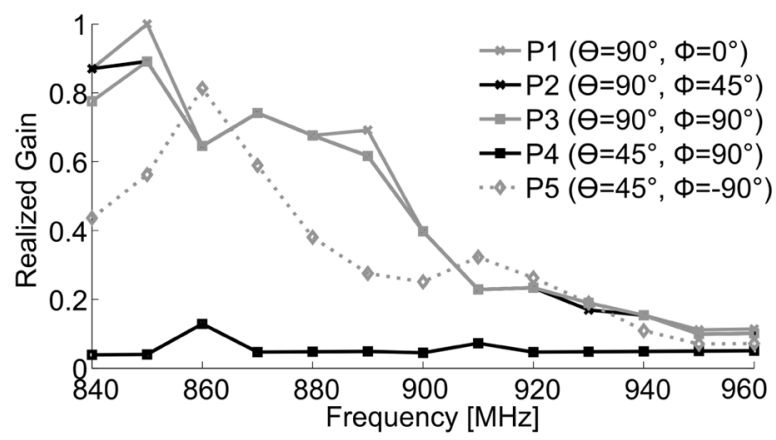

b)

Fig. 2. Single chip: (a) normalized measured analog identifier $F / F_{\max }$; (b) measured realized gain normalized by its maximum $(\tilde{G})_{\max }=0.46$. The read distance is fixed to $60 \mathrm{~cm}$ while the reader-tag orientation is changed in successive measurements according to points $P_{1} \ldots P_{5}$, as specified in the inset.

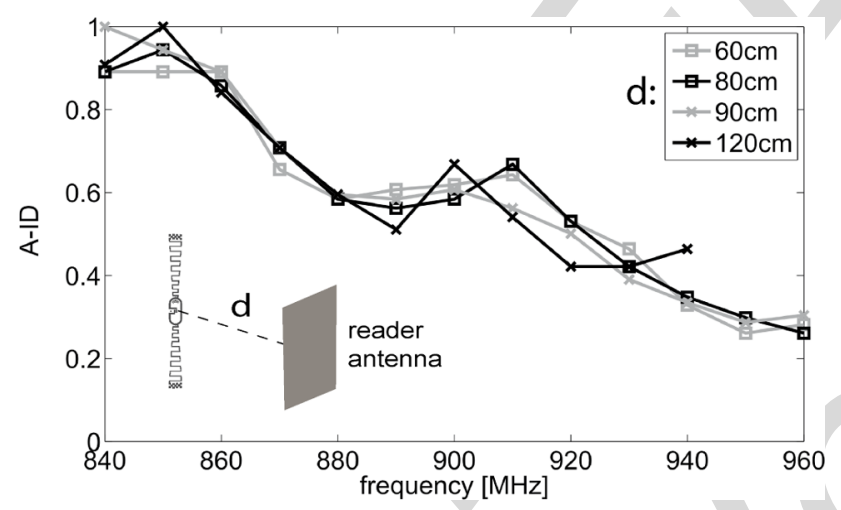

Fig. 3. Single chip: normalized A-Id measured for a single tag with variable distances from the reader and fixed angle $P_{1}:\left(\theta=90^{\circ}, \phi=0^{\circ}\right)$.

procedure, the microchip impedance can be considered quite stable over subsequent interrogations.

The phenomenology of RFID GRID systems, as theoretically found in [1], could be experimentally analyzed, under a complete control on the hardware, according to the technical solution proposed in [17], e.g., by emulating the RFID microchips with PIN diode modulators. In this paper we instead follow a more direct approach, e.g., we use commercial tags or ad-hoc designed tags embedding real RFID microchips with the purpose to recognize the RFID-grid features, theoretically argued, even in presence of all the uncertainties of electrical data.

In the following several experiments, to fully understand the true invariance of A-Id parameters, also the realized gain is measured. This is a typical parameter commonly used to characterize the radiation performance of a tag and also its interaction with time-variant materials or changing environments (humidity and gas, [18]). To be specific, the estimation of the realized gain is obtained by using the equation

$$
\tilde{G}_{n}(\theta, \phi)=\frac{p_{n}}{P_{n}^{t o} G_{R}(\theta, \phi)}\left(\frac{\lambda}{4 \pi d}\right)^{-2} .
$$

The realized gain is indeed mathematically dependent on the reader-tag mutual position and requires the knowledge of the reader's antenna gain at the specific orientation.

All the measurements are performed by an UHF ThingMagic reader, connected to a 3-dB gain linear polarized PIFA antenna. Tags under test (TUTs) are placed on a rotating platform, in order to get data acquisition at different angles. Both the reader and the tag are $110 \mathrm{~cm}$ high from the floor. It is worth noticing that the measurement scene is a real $5 \mathrm{~m}$ by $5 \mathrm{~m}$ laboratory, with no attempt to reduce the ground reflections as well as any other type of electromagnetic interaction with the surrounding objects. The measurements are repeated for 13 equally-spaced samples within the frequency band [840 MHz, $960 \mathrm{MHz}]$.

\section{ONE-CHIP TAG}

A commercial Lab-ID's UH-100 tag (Fig. 1) is here considered: a meandered dipole with a T-match impedance transformer. This tag is used to check the fingerprint invariance with the angle in a so particular one-port case. This experimentation provides a reference case for the next experiments with multiports grids, with the purpose to understand the best that can be obtained in absence of other complications such as the inter-port coupling in more complex configurations and time-varying environments.

\section{A. Analog Identifier Versus Reader-Tag Orientation}

The reader-tag distance is fixed to $60 \mathrm{~cm}$. Fig. 2(a) shows the normalized analog identifier $F(\omega) / F_{\max }$ of the considered tag obtained from the processing of the measured power at five observation angles according to (2). For comparison, Fig. 2(b) also gives the normalized realized gain in the same conditions, which, as expected by the variable gain of the tag over the vertical plane, is rather sensible to the reader-tag orientation.

The invariance of the fingerprint suggested by (3) is particularly apparent within the European RFID band, wherein the tag responds best, due to proper impedance matching.

\section{B. Analog Identifier Versus Reader-Tag Distance}

The measurements are repeated for a fixed reader-tag orientation $\left(\theta=90^{\circ}, \phi=0^{\circ}\right)$ and increasing distance $60 \mathrm{~cm}<d<$ $120 \mathrm{~cm}$. The results in Fig. 3 again show, as theoretically predicted, a substantial invariance of the analog identifier, within all the considered frequency band. 


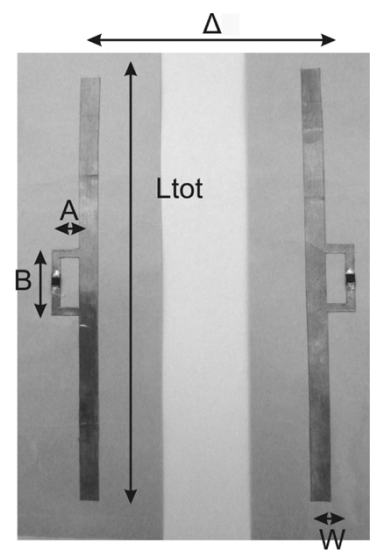

Fig. 4. Two-tags grid: prototype of equal-shape dipoles at a close distance. Size in Table I.

\section{TwO-PORTS GRIDS}

Some examples of two-port grids are here analyzed with particular attention to the improvement of the scavenging capability, obtained by means of electromagnetic coupling, and to the angle-invariance of the analog identifier. A-Id is theoretically and experimentally estimated from (2) when the reader-tag position is changed and when the grid is placed onto a timevarying object to mimic a true sensing application. In some of the following geometries the grid is composed by two close-distance dipoles having same or different size, while in a third case a true two-chip tag is analyzed.

\section{A. Scavenging-Optimized Two Tags}

In a first example, two coupled tags have been specifically designed as an interconnected system with the purpose to discuss the enhancement of RFID capabilities which may be achieved when the electromagnetic coupling is handled in a constructive way.

The tags have a conventional dipole-like layout, with a T-match impedance transformer [19] whose aspect ratio $A$, $B$ is properly selected, by the help of a finite difference time domain model of the coupled dipoles, to maximize the embedded realized gains in (1) of both the ports. A low-impedance NXP microchip transponders has been assumed, having $Z_{C}=15-j 135 \Omega$ and power sensitivity $p=-15 \mathrm{dBm}$.

The resulting antenna layout is shown in Fig. 4, and the optimized geometrical sizes are reported in Table I. The performance of the two-tags grid are here compared with that of a single port dipole having the same size as the tags of the grid except for the T-match which has been re-optimized (see again Table I) to achieve the maximum realized gain in single-port configuration.

The single tag has estimated input impedance $Z_{\text {in }}=16.3+$ $j 127$ and power transfer coefficient $\tau[$ one $-t a g]=0.94$. The antenna's realized gain $\tilde{G}_{T}$ is almost omnidirectional on the H-plane, with maximum value $\tilde{G}_{T}[o n e-t a g]=1.65$.

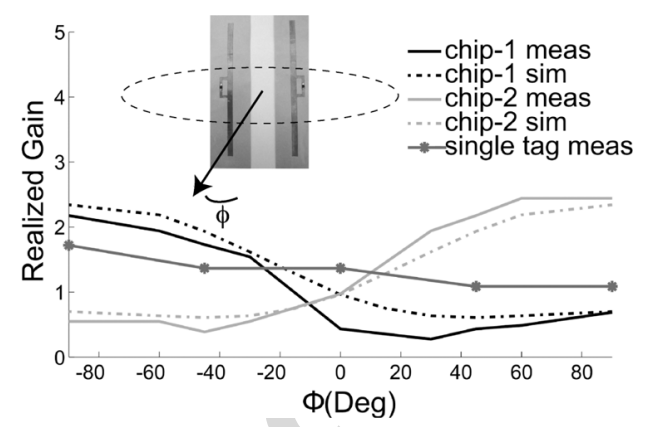

Fig. 5. Two-tags grid: measured and simulated realized gains for the coupled tags designed to improve gain performances with respect to a single tag of same size, by means of a constructive management of inter-port coupling.

The optimized two-tags grid exhibits self and mutual impedances

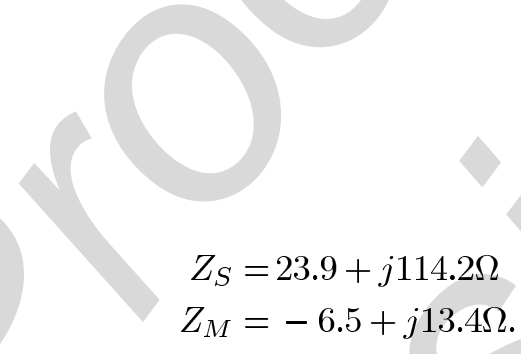

It is worth noticing the negative mutual resistance $R_{M}=$ $-5.5 \Omega$. As proved in [1], the power transfer coefficient of each port for interrogation along the normal axis of the grid is, under Hermite matching condition, $\tau_{\max }=R_{S} /\left(R_{S}+R_{M}\right)$ and may be higher than 1 provided that $R_{M}<0$, as indeed obtained in this example. For the actual case of two port grid the value $\tau[$ two - tags $]=1.37$ has been predicted by the FDTD model. The embedded realized gain of the grid, as obtained by measurements and theory, are shown in Fig. 5. Due to the proximity between the two tags, the embedded realized gains corresponding to the two ports are sensibly distorted with maximum value $\left(\tilde{G}_{1,2}\right)_{\max }=2.34$ in the endfire direction and nearly unitary gain in the broadside direction.

The measurement results are in good agreement with the simulations, verifying the theoretical analysis in [1]. The experiments moreover demonstrate that the concurrent design of the two closely spaced tags permits to improve the realized gain of each tag, with respect to the standalone dipole, of a factor

$$
\frac{\tilde{G}_{n}(\text { two }- \text { port })_{\max }}{\tilde{G}_{1}(\text { one }- \text { port })_{\max }}=1.4
$$

which definitely enlarges the maximum read distance of $20 \%$.

The measured analog identifier associated to chip 1 is shown in Fig. 6. As in the single chip case, the analog identifier is rather independent on the angle except for those frequencies where the realized gain is small, as in the case of observation angles $P_{1}(f=880 \mathrm{MHz})$ or $P_{5}(f=910 \mathrm{MHz})$. In these conditions the reader is processing a low-intensity backscattered signal and the computation in (2) is hence affected by poor accuracy. Nevertheless the measured analog identifier appears considerably 


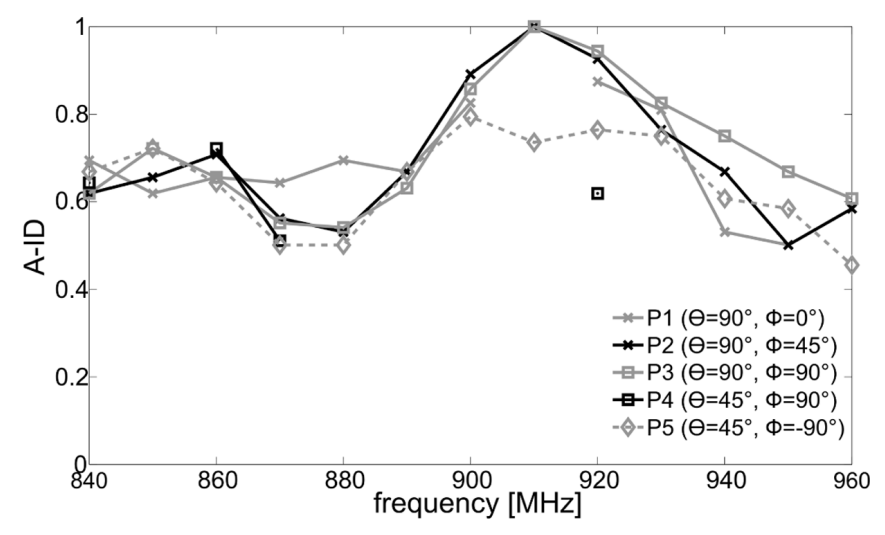

a)

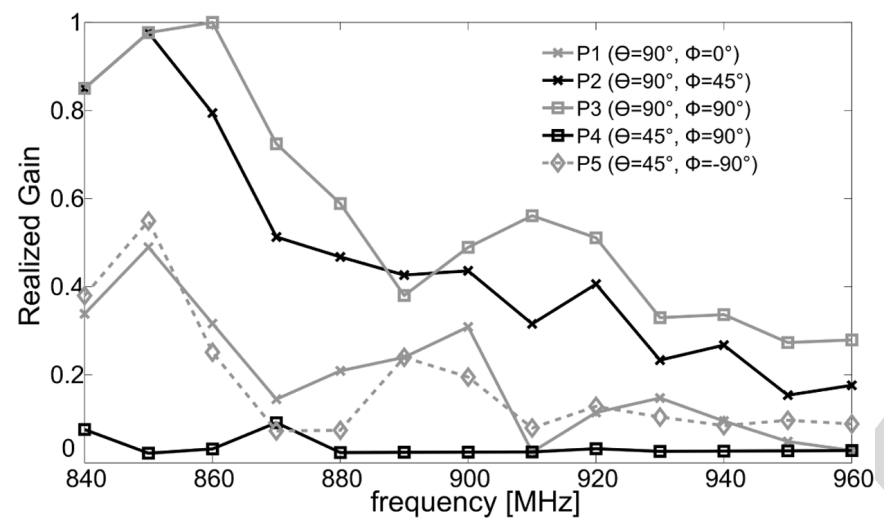

b)

Fig. 6. Two-tags grid: normalized a) A-Id and b) realized gain at some observation angles, $\left(\tilde{G}_{n}\right)_{\max }=2.34$.

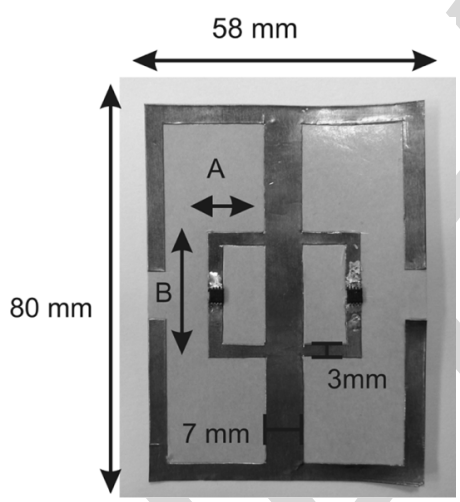

Fig. 7. Two-chip tag: prototype and size from [20].

much more stable than the measurement of the realized gain shown in Fig. 5.

\section{B. Integrated Dual-Chips Tag}

The two-port tag already presented by the authors in [20] to achieve a temperature sensor is here considered to discuss the analog identifier's invariance. The tag (Fig. 7) consists of a partly folded dipole provided with two equal-size T-match circuits to control the port impedance.

The size $(A=12 \mathrm{~mm}, B=25 \mathrm{~mm})$ of the double T-Match were designed to maximize the active power transfer coefficients $\tau_{n}=4 R_{C} R_{S} /\left|Z_{S}+Z_{C}+Z_{M}\right|^{2}$ of the two-ports grid

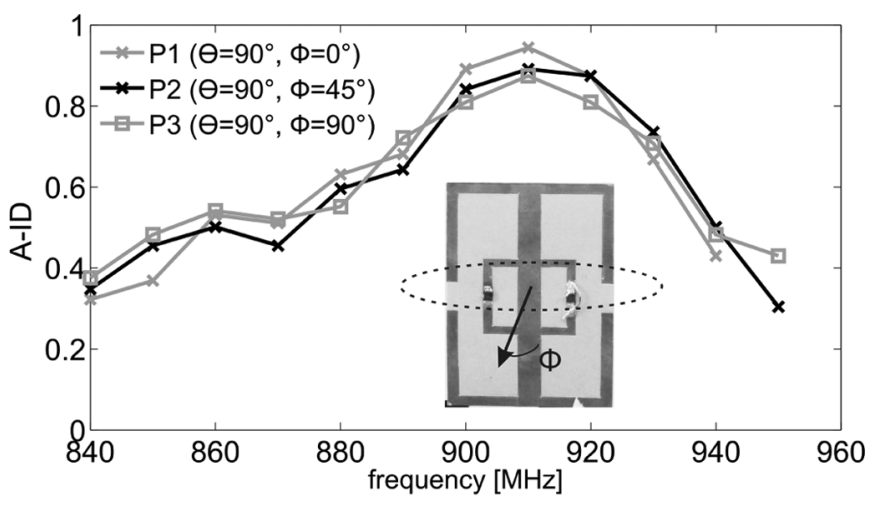

a)

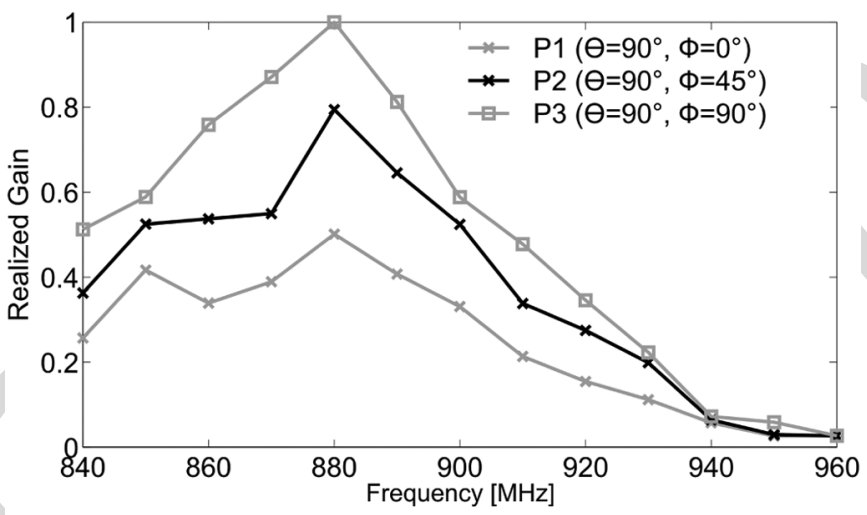

b)

Fig. 8. Two-chips Grid: normalized A-Id and realized gain of one of the ports at some observation angles. Normalization factor for the gain: $\left(\tilde{G}_{n}\right)_{\max }=0.7$.

in the broadside direction having assumed the same NXP microchip as before. An FDTD model of the antenna predicted self and mutual impedance at $870 \mathrm{MHz}$ of $Z_{S}=7.2+j 132$ and $Z_{M}=-5+j 6.25 \Omega$, respectively. Fig. 8 shows the measured analog identifiers of port 1 and the corresponding realized gain at three different observation angles over the horizontal $\left(\theta=90^{\circ}\right)$ plane. The A-Id is even more independent on the observation angle than the previous example, due to the higher values of the realized gains so that the tag will backscatter fields of similar strength, resulting in a more accurate processing of the received power.

\section{Sensing Tags}

The fingerprint-extraction procedure is here applied to the sensing of things and in particular to the detection of the physical (time-varying) state of the tagged object.

For instance, in logistics it is sometimes required to monitor the status of containers (bottles, packages, bags) with the purpose to detect variation of their solid, powder or liquid content. The self-sensing natural capabilities of RFID tags may be exploited to handle these events (see [2] for a complete introduction to RFID self-sensing). The example given here considers the sensing of sugar-powder level inside a plastic container. This is a very challenging problem for RFIDs due to the low-relative permittivity $\left(\epsilon_{r}=2.76, \sigma_{s}=2.44 \cdot 10^{-2} \mathrm{~S} / \mathrm{m}\right)$, which is very similar to that of the air $\left(\epsilon_{r}=1\right)$. The variation of filling level 


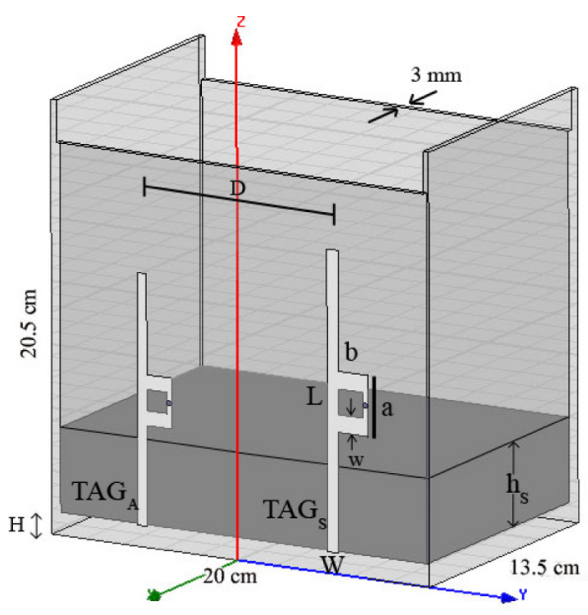

Fig. 9. Two-port grid for sensing the sugar level within a perspex box. Geometrical size (in [mm]): $H=12, D=95 ; T A G_{S} L=142.8, W=6$, $a=28.8, b=15.6, w=8.4 ; T A G_{A} L=119, W=5, a=24, b=13$ $w=7$.

is hence expected to produce only a slight modification of the tag's electromagnetic parameters, and in turn of the sensed data.

The perspex box $\left(\epsilon_{p}=2.7, \sigma_{p} \sim 0\right)$ used for the sugarpowder experiment is shown in Fig. 9. The sensing RFID Grid is now composed by two $\mathrm{T}$-match dipoles of different size attached at a close distance onto a same face of the box. The two tags, denoted as air-tag $\left(T A G_{A}\right)$ and sugar-tag $\left(T A G_{S}\right)$, have been optimized for the best conjugate matching to the NXP microchip when the box is empty $\left(\mathrm{TA}_{A}\right)$ and filled with $\operatorname{sugar}\left(T A G_{S}\right)$, respectively.

The various measurements of the analog identifiers are executed by gradually filling the box with sugar, starting from $h_{S}=0$ up to $h_{S}=14 \mathrm{~cm}$, i.e., roughly corresponding to the height of the longest dipole $T A G_{S}$. The reader and the tags are aligned to have maximum polarization efficiency.

The diagrams in Fig. 10 give the theoretical (computer simulated) and experimental analog identifier for both the tags for three different mutual orientations. The analog identifier of $T A G_{S}$ is rather insensitive (Fig. 10(a)) to the variation of the target, and could be used as a control information, e.g., it could provide the digital "label" of the object. The Analog identifier related to $T A G_{A}$ (Fig. 10(b)) is instead able to sense the sugar variation when the filling level exceeds half the tag size and spans an overall dynamics of about $50 \%$, up to saturation. The collected data are very little dependent on the mutual orientation between the reader and the container, even for rotation of $180^{\circ}$ e.g., when the two antennas are completely shadowed with respect to the reader by the sugar itself.

\section{Two DIMENSIONAL $3 \times 3$ GRID}

It is here finally considered a 3 by 3 grid (Fig. 11) made of the commercial tags already used in Section III.

The analog identifier of the central (label 22) and the edge (label 11) elements are measured by the same procedure as before. The invariance of the so obtained fingerprints (Fig. 12) respect to the reader-grid orientation is less sharp than in the previous examples, but nevertheless A-Id is still much more insensitive to rotations than the measurement of the realized gain

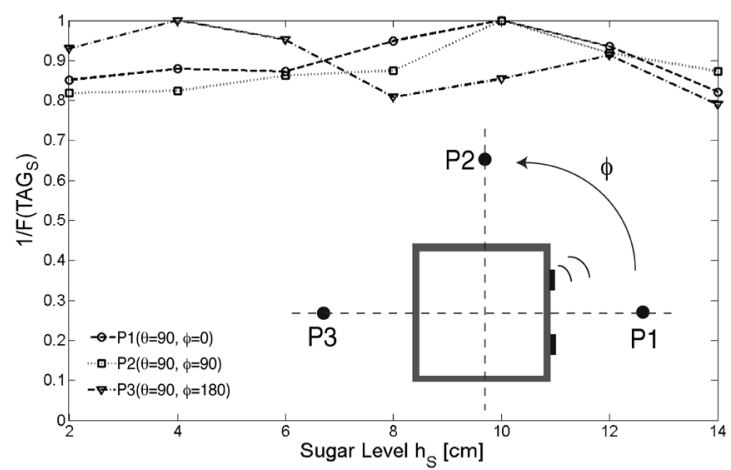

a)

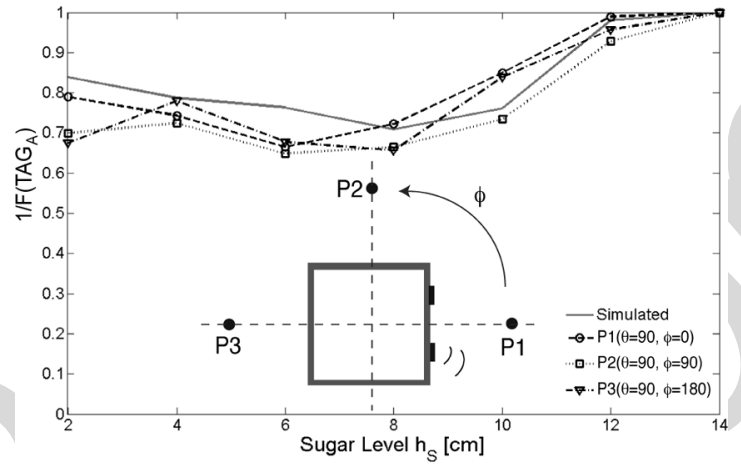

b)

Fig. 10. Two-port grid for Sugar sensing: (Normalized) analog identifiers $F_{n}^{-1}$ for: a) $T A G_{S}$ and b) $T A G_{A}$. The data acquisition has been repeated for different orientation, on the horizontal plane, between reader and tagged box so that the case of $180^{\circ}$ rotation means that the grid is completely shadowed to reader by the box.

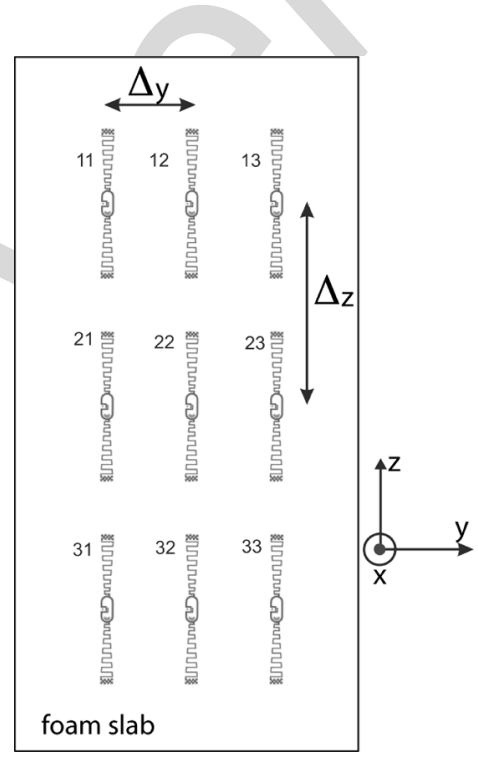

Fig. 11. $3 \times 3$ RFID Grid: the tags are attached over a foam slab. Grid steps: $\Delta_{y}=9 \mathrm{~cm}, \Delta_{z}=14 \mathrm{~cm}$.

(Fig. 13), especially when edge elements are observed. Moreover it is interesting to note that the fingerprints of the two tags are rather similar, unlike the corresponding measured gains.

\section{A. Variable Environment}

The presence of any object close to the reader-tag system can in principle modify the tag responses (turn-on power and 


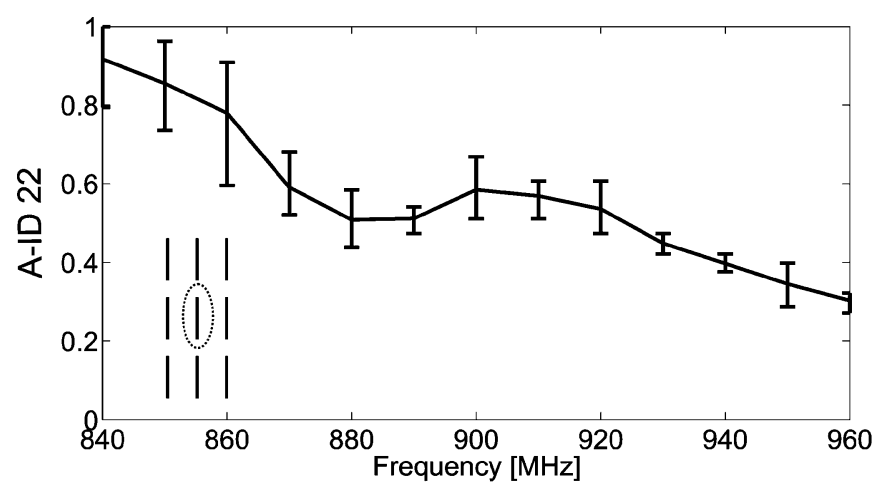

a)

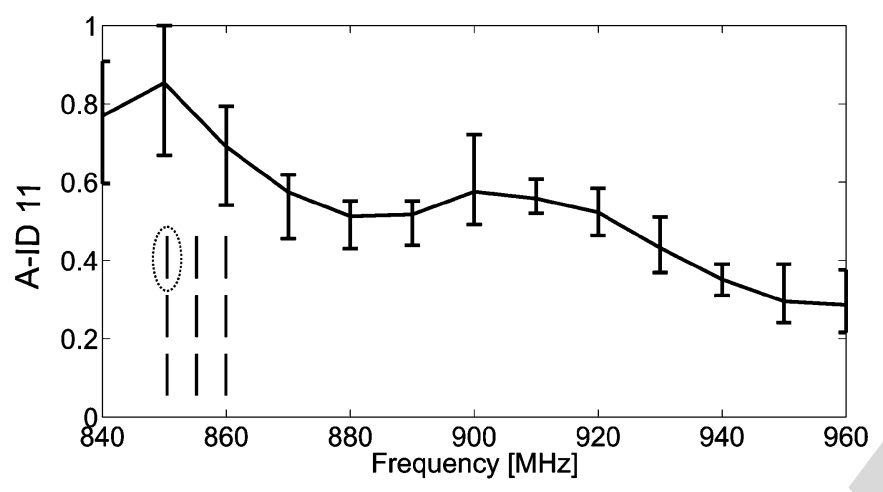

b)

Fig. 12. $3 \times 3$ RFID Grid: measured A-Id of a) central and b) edge elements. The grid plane is $80 \mathrm{~cm}$ far from the reader. The vertical segments give the residual angular variation of A-Id at each frequency sample as the observation angles are randomly chosen in the range $(\theta=90,0<\phi<2 \pi)$. The continuous line gives the angle-average fingerprint.

backscattered power) due to the electromagnetic scattering that it produces. However, since its disturbing effect applies on both the forward and backward links of the RFID communication in the same manner, it can be in principle removed by the calculation of the analog identifier which involves the processing of the turn-on and the backscattered powers.

Assuming that the scatterer is distant enough from the grid so that its input parameters are not significantly modified, the presence of the scatterer will therefore affect only the radiation pattern of the reader and of the grid. However, since the definition of A-Id drops out the reader's gain, the obtained $\left\{F_{n}\right\}$ are expected to be "transparent" to the presence of the scatterer which, in this sense, becomes "invisible" for the grid.

To verify this property, a vertical metallic cylinder, $180 \mathrm{~cm}$ high and $7 \mathrm{~cm}$ in diameter, has been placed in the region between the reader and the grid under test (Fig. 14), close to Line Of Sight condition. It was actually at a distance of $20 \mathrm{~cm}$ from the reader antenna and moved $11 \mathrm{~cm}$ sideways from the line of sight, first on the left and later on the right. The grid is measured in the fixed direction $\left(\phi=0^{\circ}, \theta=90^{\circ}\right)$.

The A-Id of the central tag is shown in Fig. 15(a) and compared to the realized gain of the same configuration $15 \mathrm{~b}$ ). This experiment is considered very challenging since the big metallic object is expected to produce a significant scattering and to seriously affect the measurement of the realized gain of grid's elements, as clearly visible in Fig. 15(b). Instead, the analog identi-

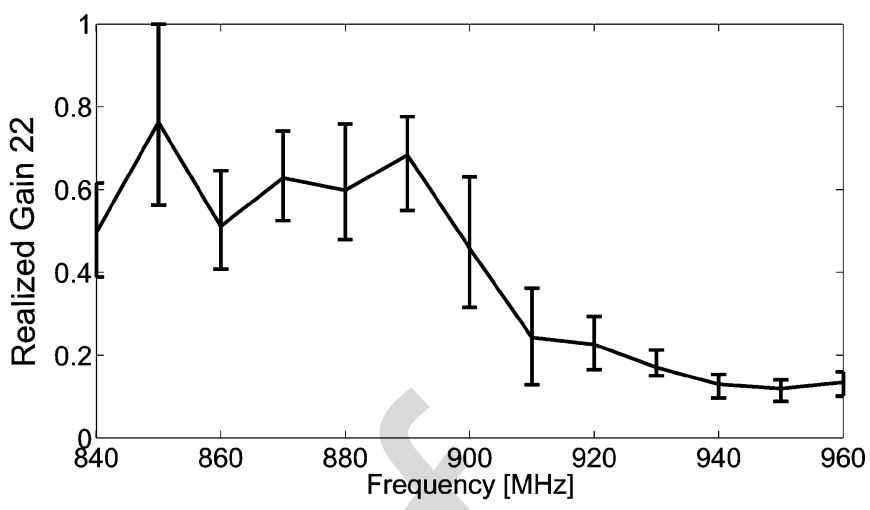

a)

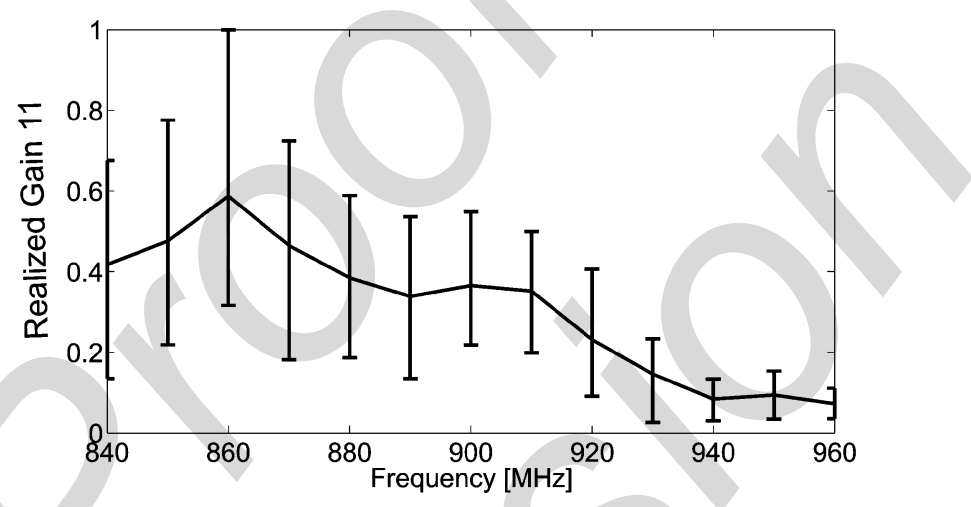

b)

Fig. 13. $3 \times 3$ RFID Grid: normalized measured realized gain of a) central element (maximum gain used for the normalization: $\tilde{G}_{22, \max }=0.29$ ) and b) edge element $\left(\tilde{G}_{11, \max }=0.53\right)$. Diagram's conventions as in Fig. 12.

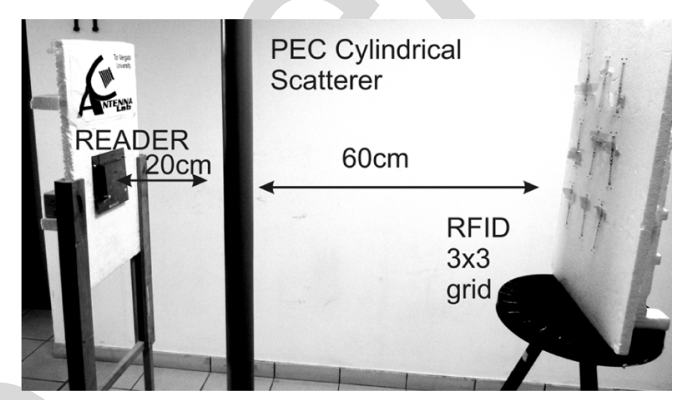

Fig. 14. $3 \times 3$ RFID Grid in variable environment: Measurement setup, comprising a RFID reader (left), the $3 \times 3$ RFID grid (right) and the metallic cylindrical scatterer interposed between the reader and the grid.

fier in Fig. 15(a) revealed also in this case to be really insensitive to the presence and to the position of the scatterer, and by extrapolation, to the motion of nearby objects or people provided that the characteristic times of such motion are large compared with the RFID interrogation rate. However, it is worth mentioning that the cancellation of the scatterer is not an exclusive property of the GRID arrangement, but it can be achieved also with just a standalone tag, as shown in the experiment in Fig. 16 where a single element of the array is placed close to the pipe scatterer as in the previous configuration.

\section{CONCLUSIONS}

The presented experimentations corroborate the theoretical analysis of RFID grids. The formulas for the active realized 


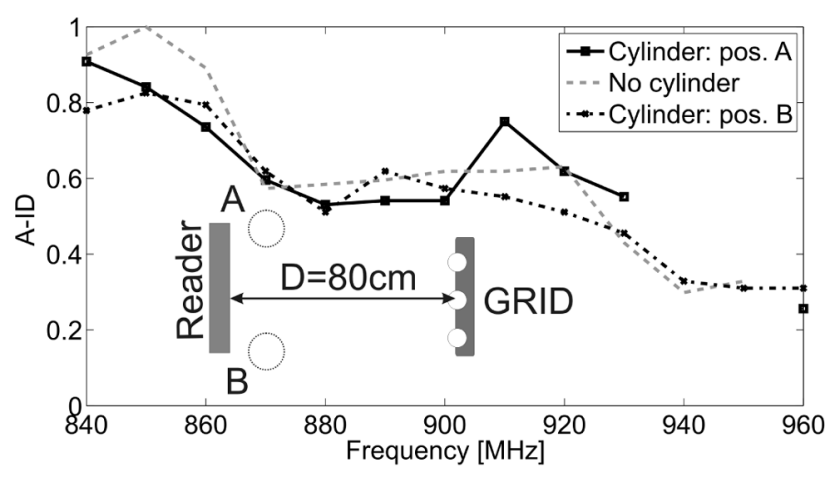

a)

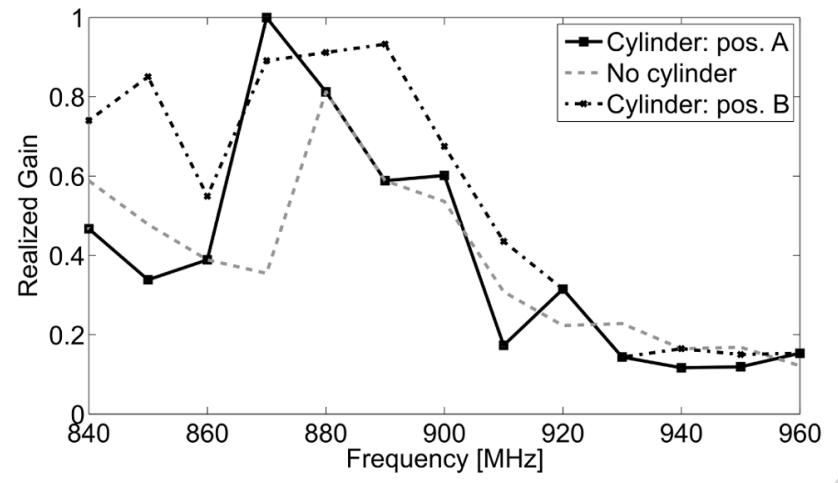

b)

Fig. 15. $3 \times 3$ RFID Grid: a cylindrical scatterer moved close to the reader/tag system into two different positions A and B: a) analog identifier of the central (22) tag collected for mutual reader-tag orientation $\phi=0^{\circ}, \theta=90^{\circ}$ and b) normalized realized gain.

gain and the impedance matching can be used together with any standard optimization design procedure. The inter-port coupling, which is generally considered as a negative effect of tags' proximity, can be instead judiciously handled to enlarge the read range of each microchip, or for the same distance, to reduce the required power.

The tag's fingerprint obtained by the calculation of the analog identifier seems to be valuable in both single and multi-chip configurations. This quantity demonstrated to be rather insensitive to the many uncertain conditions of typical RFID interrogations in a real world, e.g., to its mutual position with respect to the grid, as well as to the variation of the environment itself. However, since the definition of the fingerprint is based on the accurate measurement of the turn-on power and of the backscattered power, the true reliability of the analog identifier, for instance during sensing or security assessment, can not leave apart the quality of the reader's receiver. It is reasonable to assume that even more robust results could be achieved by using the value of the backscattered power as a quality factor of the measurements.

The cost of grid-based devices is expected to be higher than that of standard tags used as simple wireless replacement of barcodes. However multi-chips tags will offer augmented features making them closer to sensors than to radiofrequency labels.

Application to sensing appears indeed promising and in future experiments a multi-chip sensing grid could be empowered by a plurality of chemical receptors to achieve multi-variant

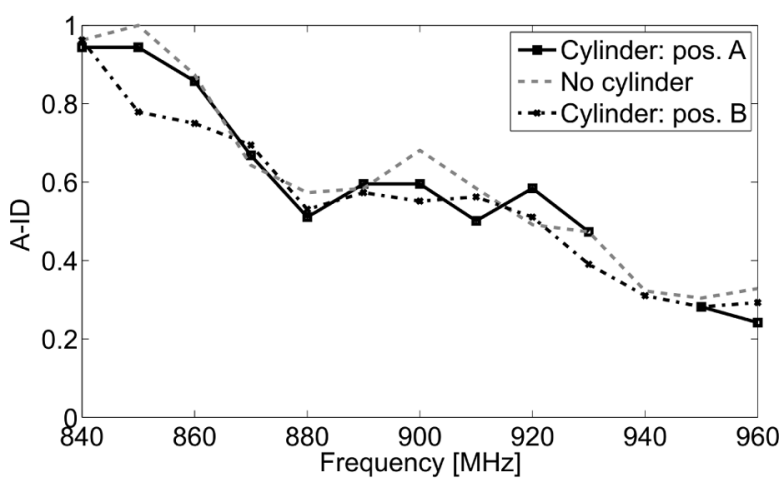

a)

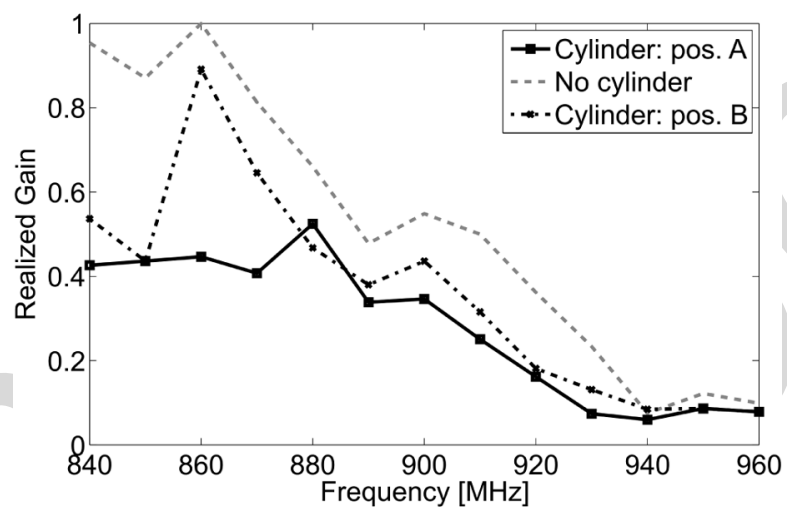

b)

Fig. 16. Single tag in presence of a variable environment in the same conditions as in the $3 \times 3$ array case. a) analog identifier b) normalized realized gain.

TABLE I

Sizes of THE Designed Single TAg AND Coupled TAgS

\begin{tabular}{c|c|c} 
& Coupled Tags $(\mathrm{mm})$ & Single Tag $(\mathrm{mm})$ \\
\hline$L_{t o t}$ & 155 & the same \\
\hline$W$ & 7 & the same \\
\hline$A$ & 10 & 16 \\
\hline$B$ & 12 & 16 \\
\hline$\triangle$ & 80 & -
\end{tabular}

low-cost remote sensors. The fingerprint concept will give a reasonable confidence that a change in the grid's responses in successive interrogations will be related to a change of the physical parameters under test, rather than to a change of the mutual reader-tag position. So, there will be many opportunities to develop new devices but also there will be the need of ad-hoc data retrieval algorithms to master specific applications.

\section{REFERENCES}

[1] G. Marrocco, "RFID grids: Part I-Electromagnetic theory," IEEE Trans. Antennas Propag..

[2] G. Marrocco, L. Mattioni, and C. Calabrese, "Multi-port sensor RFIDs for wireless passive sensing-Basic theory and early simulations," IEEE Trans. Antennas Propag., vol. 56, no. N.8, pp. 2691-2702, Aug. 2008.

[3] M. Philipose, J. R. Smith, B. Jiang, A. Mamishev, S. Roy, and K. Sundara-Rajan, "Battery-free wireless identification and sensing," IEEE Perv. Comput., vol. 4, no. N.1, pp. 37-45, Jan.-Mar. 2005.

[4] P. Nikitin, K. V. S. Rao, and S. Lam, "RFID Tags With Enhanced Range and Bandwidth Obtained by Spatial Antenna Diversity," U.S. Patent no. US2009/ 0219158, Sep. 2008. 
[5] J. W. Wallace and M. A. Jensen, "Mutual coupling in MIMO wireless systems: A rigorous network theory analysis," IEEE Trans. Wireless Commun., vol. 3, no. N.4, pp. 1317-1325, Jul. 2004.

[6] D. Preuveneers and Y. Berbers, "Internet of things: A context- awareness perspective," in The Internet of Things: From RFID to the NextGeneration Pervasive Networked Systems, L. Yan, Y. Zhang, L. T. Yang, and H. Ning, Eds. London: Auerbach Publications, 2008.

[7] L. Ukkonen and L. Sydanheimo, "Threshold power-based radiation pattern measurement of passive UHF RFID tags," in Proc. PIERS, Cambridge, 2010, pp. 87-90.

[8] P. V. Nikitin and K. V. S. Rao, "Theory and measurement of backscattering from RFID tags," IEEE Antennas Propag. Mag., vol. 48, no. N. 6, pp. 5459-5462, Dec. 2006.

[9] S. Skali, C. Chantepy, and S. Tedjini, "On the measurement of the delta radar cross section ( $\triangle \mathrm{RCS})$ for UHF tags," in Proc. IEEE Int. Conf. on RFID 2009, pp. 346-351.

[10] S. L. Chen, K.-H. Lin, and R. Mittra, "A measurement technique for verifying the match condition of assembled RFID tags," IEEE Trans. Instrum. Meas., vol. 59, no. 8, Aug. 2010.

[11] J. P. Daniel, "Mutual coupling between antennas for emission and reception- application to passive and active dipoles," IEEE Antennas Propag. Mag., vol. 22, no. 2, pp. 347-349, 1974.

[12] F. Lu, X. Chen, and T. T. Ye, "Performance analysis of stacked RFID tags," in Proc. IEEE Int. Conf. on RFID, 2009, pp. 330-337.

[13] K. Lee and T. Chu, "Mutual coupling mechanisms within arrays of nonlinear antennas," IEEE Trans. Electromagn. Compat., vol. 47, no. 4, pp. 963-970, 2005.

[14] X. Chen, F. Lu, and T. T. Ye, "The weak spots in stacked UHF RFID tags in NFC applications," in Proc. IEEE Int. Conf. on RFID, 2010, pp. $181-186$.

[15] V. Rizzoli, A. Costanzo, M. Rubini, and D. Masotti, "Rigorous investigation of interactions between passive RFID tags by means of nonlinear/electromagnetic co-simulation," in Proc. Eur. Microwave Conf., 2006, pp. 722-725.

[16] Y. Tanaka, Y. Umeda, O. Takyu, M. Nakayama, and K. Kodama, "Change of read range for UHF passive RFID tags in close proximity," in Proc. IEEE Int. Conf. on RFID, 2009, pp. 338-345.

[17] J. D. Griffin and G. D. Durgin, "Complete link budgets for backscatterradio and RFID systems," IEEE Antennas Propag. Mag., vol. 51, no. N.2, pp. 11-25, 2009.

[18] J. Virtanen, L. Ukkonen, T. Bjorninen, and L. Sydanheimo, "Printed humidity sensor for UHF RFID systems," IEEE Sensors Applicat., pp. 269-272, 2010

[19] G. Marrocco, "The art of UHF RFID antenna design: Impedance matching and size-reduction techniques," IEEE Antennas Propag. Mag., vol. 50, no. N.1, pp. 66-79, Feb. 2008.

[20] S. Caizzone, C. Occhiuzzi, and G. Marrocco, "Multi-chip RFID antenna integrating shape-memory alloys for detection of thermal thresholds," IEEE Trans. Antennas Propag., to be published.

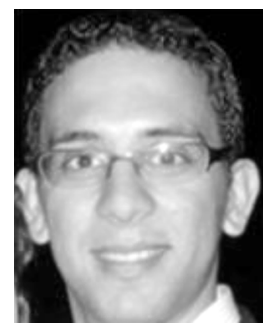

tennas.

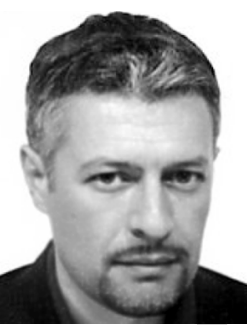

Gaetano Marrocco was born in Teramo, Italy, on August 29, 1969. He received the Laurea degree in electronic engineering (Laurea cum laude and Academic Honour) and the Ph.D. degree in applied electromagnetics from the University of L'Aquila, Italy, in 1994 and 1998, respectively.

Since 1997, he has been a Researcher at the University of Rome "TorVergata," Rome, Italy, where he currently teaches Antenna Design and Medical Radio-Systems, manages the Antenna Lab and is Advisor in the Geo-Information Ph.D. program. In October 2010, he achieved the level of Associate Professor of electromagnetic. In summer 1994, he was at the University of Illinois at Urbana-Champain as a Postgraduate student. In autumn 1999, he was a Visiting Researcher at the Imperial College in London, U.K. In 2008, he joined the Ph.D. program of the University of Grenoble (FR). His research is mainly directed to the modeling and design of broad band and ultrawideband (UWB) antennas and arrays as well as of sensor-oriented miniaturized antennas for biomedicine, aeronautics and radiofrequency identification (RFID). He has been involved in several Space, Avionic, Naval and Vehicular programs of the European Space Agency, NATO, Italian Space Agency, and the Italian Navy about the analysis and the design of non conventional antennas and systems. He holds eight patents on broadband naval antennas and structural arrays, and on sensor RFID systems.

Prof. Marrocco currently serves as an Associate Editor of the IEEE Antennas and Wireless Propagation Letters, he is a reviewer for the IEEE TRANSACTION on ANTENNAS AND PROPAGATION, IEEE PROCEEDINGS, IEEE TRANSACTIONS ON MICROWAVE THEORY AND TECHNIQUES, and is a member of Technical Program Committee of several International Conferences. In 2008, he was the General Chairman of the first Italian multidisciplinary scientific workshop on RFID: RFIDays-2008: Emerging Technology for Radiofrequency Identification. He was the Co-Chair of the RFIDays-2010 International Workshop in Finland and Chairman of the Local Committee of the V European Conference on Antennas and Propagation.

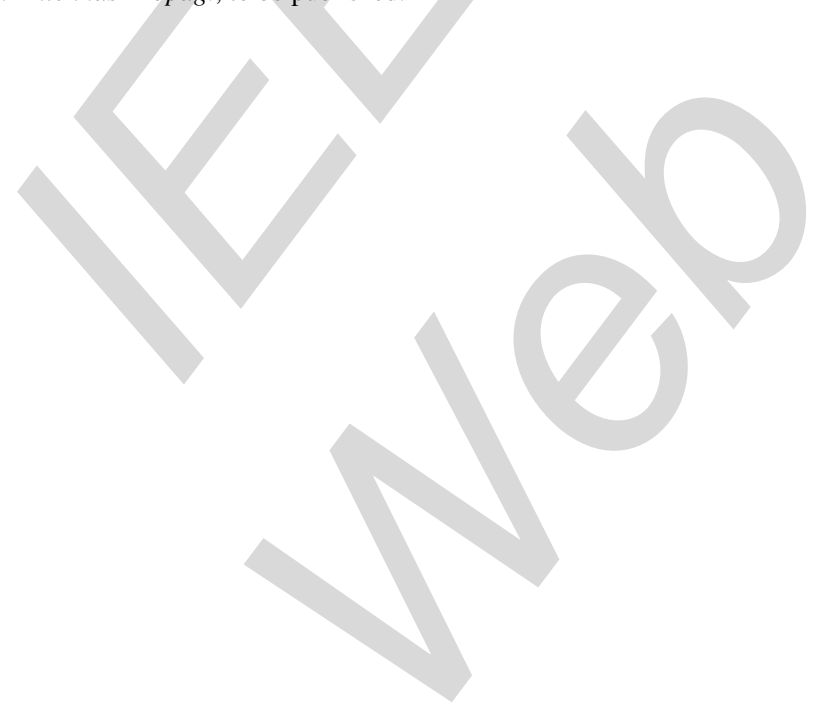




\title{
RFID Grids: Part II—Experimentations
}

\author{
Stefano Caizzone and Gaetano Marrocco
}

\begin{abstract}
The RFID Grid is a model for generally coupled multitudes of tags including single-chip tags in close mutual proximity or a single tag with a plurality of embedded microchips. Some properties of this new entity, useful for passive Sensing and for Security, are the possibility to increase the read-range and to provide responses rather insensitive to the interrogation modalities. These recently introduced issues are here experimented for the first time with many real-world examples comprising multi-chip configurations designed for improved power scavenging and for passive sensing of things.
\end{abstract}

Index Terms-Array, grid, MIMO, remote sensing, RFID, sensor, space diversity.

\section{INTRODUCTION}

V ERY recently, the paper [1] introduced the concept of RFID Grid, e.g., a generally coupled multitude of UHF (or higher frequency) RFID tags, including single-chip tags in close mutual proximity or a single tag with a plurality of embedded microchips. This model is useful to predict the impedance and the backscattering performances of tags over a dense collection of objects such as items over a shelf, as well as to design new sensing-oriented tags. RFID tags, indeed, may be equipped with more than a single chip to communicate the identity of the object, by one of the chip, and its physical state by means of the other chip or a combination of both [2]-[4].

In [1] the basic equations for the direct and inverse links were derived by using the very general model of multi-port loaded scatterers. The theoretical analysis has permitted to discover new features, some of them common to MIMO and Space-Diversity systems [5], and in particular the possibility

i) to handle the inter-antenna coupling in a useful way with the purpose to improve the power harvested by the microchip and hence to enlarge the read range;

ii) to achieve measured analog backscattered information which are rather independent on the mutual position between reader and tags themselves (distance and orientation). Such a function, denoted as Analog Identifier (A-Id) as complementation of the Digital Identifier (D-Id) normally stored in the microchip memory, originates from the combined processing of direct- and

Manuscript received October 16, 2010; revised December 14, 2010; accepted January 24, 2011. Date of publication June 09, 2011; date of current version August 03, 2011.

S. Caizzone was with the DISP-University of Roma Tor Vergata Via del Politecnico, 1, 00133 Roma, Italy . He is now with the Antenna Group, Institute of Communications and Navigation, German Space Agency (DLR), 82230 Wessling, Germany.

G. Marrocco is with the DISP-University of Roma Tor Vergata Via del Politecnico, 1, 00133 Roma, Italy (e-mail: marrocco@disp.uniroma2.it).

Color versions of one or more of the figures in this paper are available online at http://ieeexplore.ieee.org.

Digital Object Identifier 10.1109/TAP.2011.2158974 inverse-link measurements. A-Id could have significant application in the context of sensing and security. In both cases, indeed, the analog response of the tag (basically the level of the backscattered power or the turn-on power) is processed in addition to the digital microchip identifier, to earn some physical information about the tag's identity (security) and about the variation of the tagged object of the nearby environment (sensing). At these purposes it is of primary importance that the reading of analog information from the tag is as more independent as possible on the interrogation modality, in order to easily collect the tag's response at different times without the need to strictly replicate a specific reader-tag set-up. In particular, the availability of reading-robust functions could boost the emerging pervasive sensing and context aware applications within the Internet of Things paradigm [6].

The wireless characterization of RFID tags has been mainly focused to the measurement of the tag's read range or radiation patterns starting from the experimental evaluation of the threshold (or turn-on) power level [7] of the tag. Another performance parameter, commonly measured, is the backscattered power from the tag to the reader's detector or, more in general, the tag's radar cross section [8], [9]. Both turn-on power and RCS are related to gain and input impedance of the tag and therefore they cannot be easily de-embedded by means of conventional wireless measurements techniques. The characterization of tag input impedance is instead generally performed by using balanced probes and de-embedding procedures as in [10].

The response of RFID grids has never been characterized in a systematic way: some studies [11]-[16] have been conducted in order to assess the overall influence of mutual coupling between tags over the degradation of the RFID link and the results are mainly aiming at mitigating this degradation (see [1] for an extended discussion).

On the other hand, in the perspective of understanding the coupling between tags to exploit useful features, this paper presents a detailed experimental campaign to prove and discuss some of the concepts introduced in the previous companion paper [1] by the help of real-world examples having incremental complexity. Starting from a tag with a single microchip, the experiments go on through integrated two-microchips tags, up to $3 \times 3$ RFID grids. Measurements have been performed in presence of different reader-grid positions, time-varying tagged objects and non-stationary environments. The aim is to demonstrate that the RFID-oriented analytical formulas provided in [1] can be useful to dominate the inter-port coupling within the design task and to verify the true degree of invariance of the analog identifier in uncontrolled conditions.

The paper is organized as follows. Section II introduces the measurement procedures for the relevant RFID responses, 
including angle- and distance-invariant analog identifiers, the turn-on power and the realized gain. A single-chip tag in standalone configuration is measured in Section III to understand the quality of responses achievable in absence of inter-tag interactions and of other ambient perturbation. Three examples of two-chips grids are then considered in detail in Section IV, with particular attention to evaluate the improvement in the RFID performances by a systematic design of the grid, and to characterize the angular invariance of the analog identifier also in sensing-like applications. Finally, a $3 \times 3$ grid of commercial tags is explored in Section V where the sensitivity of the fingerprint is experienced with respect to the presence of metallic scatterers close to the interrogating reader.

\section{BACKGROUND AND MEASUREMENT METHODOLOGY}

According to [1], the power scavenging performances of an RFID Grid are completely characterized in the free space by means of the embedded realized gain of each $n$th port

$$
\tilde{G}_{n}=4 \eta_{0} R_{C, n}\left|\left[\mathbf{Y}_{G}\right]_{n} \cdot \mathbf{g}\right|^{2}
$$

where $\left[\mathbf{Y}_{G}\right]_{n}$ is the $n$th row of the admittance matrix $\mathbf{Y}_{\mathbf{G}}=$ $\left(\mathbf{Z}_{C}+\mathbf{Z}\right)^{-1}$ of the grid, being $\mathbf{Z}$ the impedance matrix referred to the RFID microchips' connections, and $\mathbf{Z}_{C}$ a diagonal matrix containing the equivalent impedances $\left\{Z_{C, n}=R_{C, n}+\right.$ $\left.j X_{C, n}\right\}$ of the microchips. The vector $\mathrm{g}$ of elements $g_{n}=$ $\sqrt{R_{n n} G_{n}(\underline{\hat{r}}) \chi_{n} / \eta_{0}} e^{j \Phi_{n}(\underline{\hat{r}})}$ embeds all the electromagnetic parameters associated with the ports, e.g., the open circuit gain $G_{n}$, the polarization mismatch $\chi_{n}$, the self resistance $R_{n n}$ and finally the phase $\Phi_{n}$ of the embedded field. The gain $\tilde{G}_{n}$ is the parameter to be maximized (in the useful direction) for best power scavenging and hence for longest read distance.

Physical information about the Grid and about its interaction with the tagged objects can be extracted from the analog identifier or fingerprint $F_{n}$ of the $n$th port, independently on the reading modalities. The analog identifier has been defined in [1] as a function of the turn-on power $P_{n}^{t o}$ of the $n$th port and of the corresponding backscattered power $P_{R \leftarrow T n}$ collected by the reader. For the particular modulation scheme for which the microchip' impedance is switched between a high impedance and the scavenging impedance $Z_{C, n}$, the analog identifier may be written in a very compact form

$$
F_{n} \equiv \frac{p_{n}}{\sqrt{P_{R \leftarrow T n} P_{n}^{t o}}} \propto R_{C, n}\left|Y_{G, n n}\right|
$$

where $p_{n}$ is the sensitivity of the microchip at the $n$th port, and the proportionality factor accounts for the reader's front-end and the modulation scheme, as discussed later on.

The particular case of the single-chip tag yields

$$
F=\propto \frac{R_{C}}{\left|Z_{A}+Z_{C}\right|}
$$

where $Z_{A}$ is simply the input impedance of the antenna of the tag. Both the above expressions may be evaluated along with the frequency to carry on further informations.
The physical meaning of analog identifier, and its calculation from experimental data deserves some clarification. It combines, by definition, measured data from both the direct (power harvesting) and reverse (power backscattering) links. Under the hypothesis of a stationary environment between the reader's interrogation and the tag's responses, any interaction with the nearby environment, and the mutual distance and orientation between the reader and the RFID systems, will affect both the links in a same manner, thanks to the antenna reciprocity. Hence, it was demonstrated that the simple combination of turn-on power and backscattered power will mathematically remove any kind of the above mentioned electromagnetic interactions. What remains is the functional dependence on the $n$th diagonal entry $Y_{G, n n}$ of the complete admittance matrix, in the case of multichip systems, or the dependence on the series connection between the input impedance of the tag's antenna and of the microchip in the simpler case of one-chip tag. In this sense, the A-Id is a structural property of the (multi-port) tag. Since the network admittance matrix is sensitive to the object where the tag is attached on, the variation of the A-Id in successive measurements may be related to time-changing chemical-physical properties of the object, therefore giving sensing capability. The orientation-invariance of A-Id will permit to perform this measurement without taking care to replicate a same reader-tag position at successive times.

The calculation of A-Id requires the knowledge of the turn-on power $P_{n}^{t o}$. At this purpose the power entering into the reader's antenna is gradually increased, in a controlled way, till the $n$th chip begins responding. At that moment, such a value of input power is the required turn-on $P_{i n}^{t o}$ and the corresponding backscattered power $P_{R \leftarrow T n}$ received by the reader is stored on a Personal Computer. In case of multi-chips configurations, an inventory task is preliminary performed, e.g., all the chips' identifiers are collected, and then each chip is interrogated sequentially.

Usually, commercial readers return backscattered power in the form of RSSI (received signal strength indication), after a process of demodulation, frequency conversion and equalization. The particular relationship between $P_{R \leftarrow T n}$ and the RSSI is hardware-dependent but often takes the form

$$
P_{R \leftarrow T n}(d B)=K_{1} R S S I+K_{2}
$$

with $K_{1,2}$ are specific to the reader's chipset but also to the modulation scheme. In all the measurements shown in this work, the stored RSSI signal is related to the high-level modulation state. Anyway, the experimentally-evaluated A-Id from (2) and (4) is not an absolute fingerprint of the tag but it is filtered by the reader's circuitry. The use of A-Id for sensing will therefore requires a same reader family for subsequent interrogations to avoid other kinds of uncertainties.

Finally, the A-Id depends on the microchips' impedance that, as its is well known, exhibits a non-linear dependence on the impinging interrogation power. The proposed procedure to derive the A-Id, however, enforces the turn-on power condition: it means that the microchip will always receive the same amount of power, just corresponding to its sensitivity. Thanks to this 


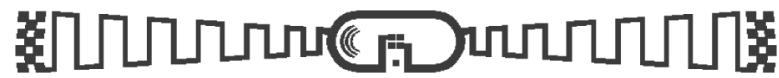

Fig. 1. The LabID UHF tag used in the experimentation campaign.

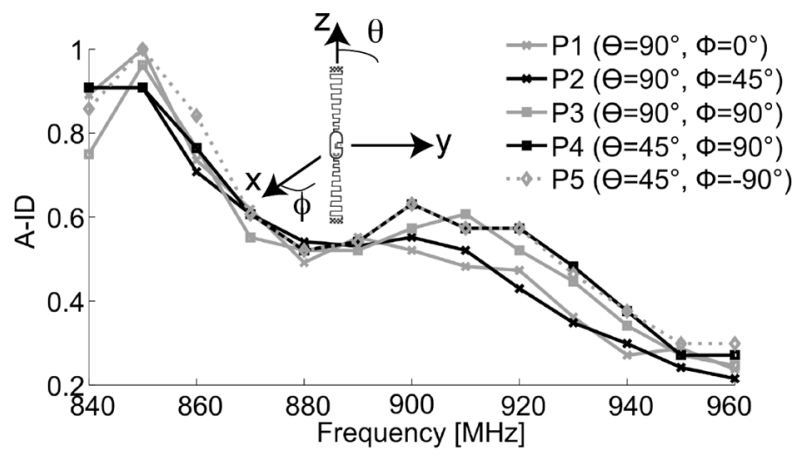

a)

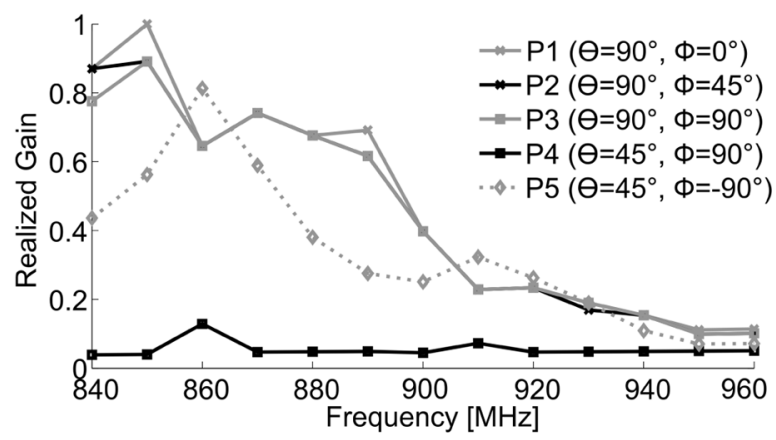

b)

Fig. 2. Single chip: (a) normalized measured analog identifier $F / F_{\max }$; (b) measured realized gain normalized by its maximum $(\tilde{G})_{\max }=0.46$. The read distance is fixed to $60 \mathrm{~cm}$ while the reader-tag orientation is changed in successive measurements according to points $P_{1} \ldots P_{5}$, as specified in the inset.

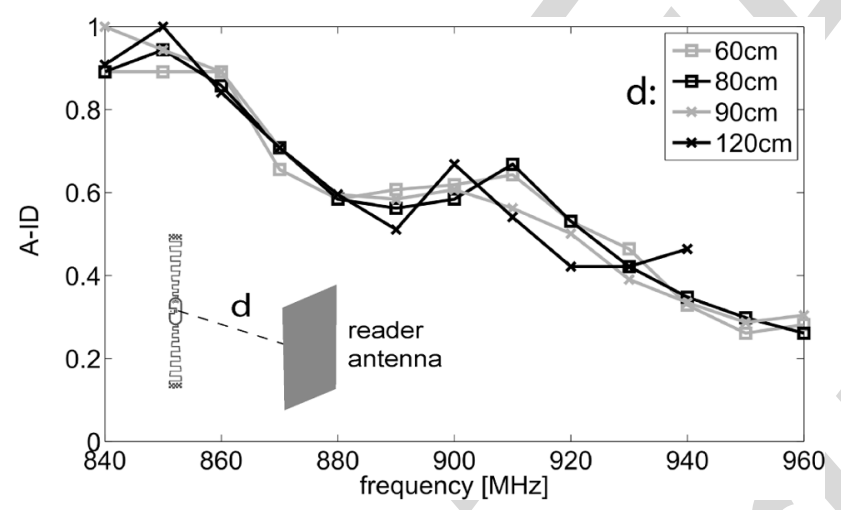

Fig. 3. Single chip: normalized A-Id measured for a single tag with variable distances from the reader and fixed angle $P_{1}:\left(\theta=90^{\circ}, \phi=0^{\circ}\right)$.

procedure, the microchip impedance can be considered quite stable over subsequent interrogations.

The phenomenology of RFID GRID systems, as theoretically found in [1], could be experimentally analyzed, under a complete control on the hardware, according to the technical solution proposed in [17], e.g., by emulating the RFID microchips with PIN diode modulators. In this paper we instead follow a more direct approach, e.g., we use commercial tags or ad-hoc designed tags embedding real RFID microchips with the purpose to recognize the RFID-grid features, theoretically argued, even in presence of all the uncertainties of electrical data.

In the following several experiments, to fully understand the true invariance of A-Id parameters, also the realized gain is measured. This is a typical parameter commonly used to characterize the radiation performance of a tag and also its interaction with time-variant materials or changing environments (humidity and gas, [18]). To be specific, the estimation of the realized gain is obtained by using the equation

$$
\tilde{G}_{n}(\theta, \phi)=\frac{p_{n}}{P_{n}^{t o} G_{R}(\theta, \phi)}\left(\frac{\lambda}{4 \pi d}\right)^{-2} .
$$

The realized gain is indeed mathematically dependent on the reader-tag mutual position and requires the knowledge of the reader's antenna gain at the specific orientation.

All the measurements are performed by an UHF ThingMagic reader, connected to a 3-dB gain linear polarized PIFA antenna. Tags under test (TUTs) are placed on a rotating platform, in order to get data acquisition at different angles. Both the reader and the tag are $110 \mathrm{~cm}$ high from the floor. It is worth noticing that the measurement scene is a real $5 \mathrm{~m}$ by $5 \mathrm{~m}$ laboratory, with no attempt to reduce the ground reflections as well as any other type of electromagnetic interaction with the surrounding objects. The measurements are repeated for 13 equally-spaced samples within the frequency band [840 MHz, $960 \mathrm{MHz}]$.

\section{ONE-CHIP TAG}

A commercial Lab-ID's UH-100 tag (Fig. 1) is here considered: a meandered dipole with a T-match impedance transformer. This tag is used to check the fingerprint invariance with the angle in a so particular one-port case. This experimentation provides a reference case for the next experiments with multiports grids, with the purpose to understand the best that can be obtained in absence of other complications such as the inter-port coupling in more complex configurations and time-varying environments.

\section{A. Analog Identifier Versus Reader-Tag Orientation}

The reader-tag distance is fixed to $60 \mathrm{~cm}$. Fig. 2(a) shows the normalized analog identifier $F(\omega) / F_{\max }$ of the considered tag obtained from the processing of the measured power at five observation angles according to (2). For comparison, Fig. 2(b) also gives the normalized realized gain in the same conditions, which, as expected by the variable gain of the tag over the vertical plane, is rather sensible to the reader-tag orientation.

The invariance of the fingerprint suggested by (3) is particularly apparent within the European RFID band, wherein the tag responds best, due to proper impedance matching.

\section{B. Analog Identifier Versus Reader-Tag Distance}

The measurements are repeated for a fixed reader-tag orientation $\left(\theta=90^{\circ}, \phi=0^{\circ}\right)$ and increasing distance $60 \mathrm{~cm}<d<$ $120 \mathrm{~cm}$. The results in Fig. 3 again show, as theoretically predicted, a substantial invariance of the analog identifier, within all the considered frequency band. 


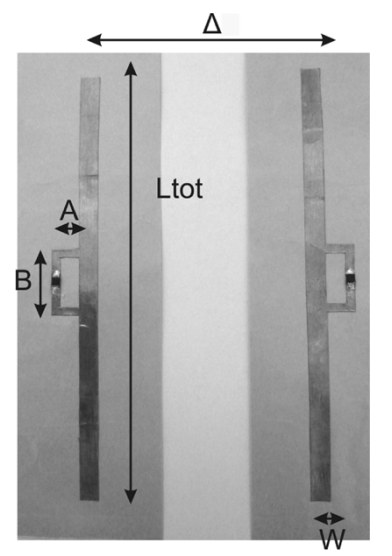

Fig. 4. Two-tags grid: prototype of equal-shape dipoles at a close distance. Size in Table I.

\section{TwO-PORTS GRIDS}

Some examples of two-port grids are here analyzed with particular attention to the improvement of the scavenging capability, obtained by means of electromagnetic coupling, and to the angle-invariance of the analog identifier. A-Id is theoretically and experimentally estimated from (2) when the reader-tag position is changed and when the grid is placed onto a timevarying object to mimic a true sensing application. In some of the following geometries the grid is composed by two close-distance dipoles having same or different size, while in a third case a true two-chip tag is analyzed.

\section{A. Scavenging-Optimized Two Tags}

In a first example, two coupled tags have been specifically designed as an interconnected system with the purpose to discuss the enhancement of RFID capabilities which may be achieved when the electromagnetic coupling is handled in a constructive way.

The tags have a conventional dipole-like layout, with a T-match impedance transformer [19] whose aspect ratio $A$, $B$ is properly selected, by the help of a finite difference time domain model of the coupled dipoles, to maximize the embedded realized gains in (1) of both the ports. A low-impedance NXP microchip transponders has been assumed, having $Z_{C}=15-j 135 \Omega$ and power sensitivity $p=-15 \mathrm{dBm}$.

The resulting antenna layout is shown in Fig. 4, and the optimized geometrical sizes are reported in Table I. The performance of the two-tags grid are here compared with that of a single port dipole having the same size as the tags of the grid except for the T-match which has been re-optimized (see again Table I) to achieve the maximum realized gain in single-port configuration.

The single tag has estimated input impedance $Z_{\text {in }}=16.3+$ $j 127$ and power transfer coefficient $\tau[$ one - tag $]=0.94$. The antenna's realized gain $\tilde{G}_{T}$ is almost omnidirectional on the H-plane, with maximum value $\tilde{G}_{T}[o n e-t a g]=1.65$.

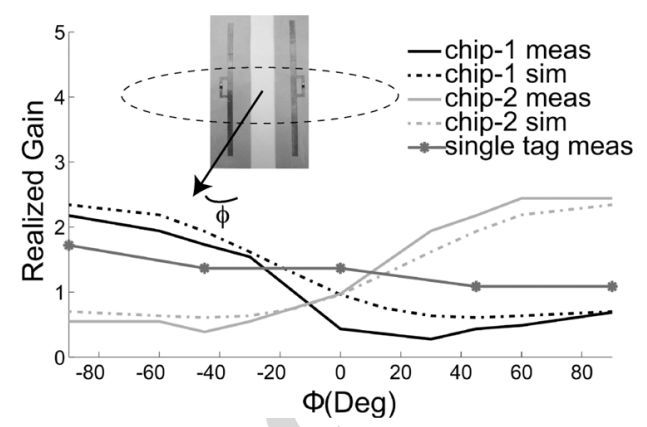

Fig. 5. Two-tags grid: measured and simulated realized gains for the coupled tags designed to improve gain performances with respect to a single tag of same size, by means of a constructive management of inter-port coupling.

The optimized two-tags grid exhibits self and mutual impedances

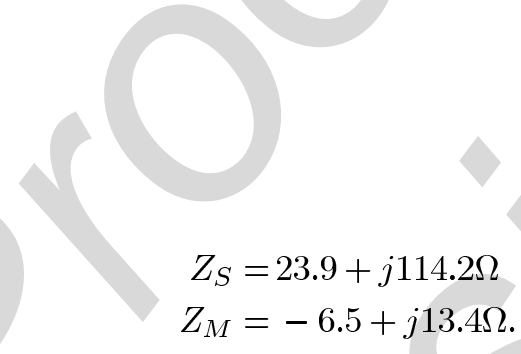

It is worth noticing the negative mutual resistance $R_{M}=$ $-5.5 \Omega$. As proved in [1], the power transfer coefficient of each port for interrogation along the normal axis of the grid is, under Hermite matching condition, $\tau_{\max }=R_{S} /\left(R_{S}+R_{M}\right)$ and may be higher than 1 provided that $R_{M}<0$, as indeed obtained in this example. For the actual case of two port grid the value $\tau[$ two - tags $]=1.37$ has been predicted by the FDTD model. The embedded realized gain of the grid, as obtained by measurements and theory, are shown in Fig. 5. Due to the proximity between the two tags, the embedded realized gains corresponding to the two ports are sensibly distorted with maximum value $\left(\tilde{G}_{1,2}\right)_{\max }=2.34$ in the endfire direction and nearly unitary gain in the broadside direction.

The measurement results are in good agreement with the simulations, verifying the theoretical analysis in [1]. The experiments moreover demonstrate that the concurrent design of the two closely spaced tags permits to improve the realized gain of each tag, with respect to the standalone dipole, of a factor

$$
\frac{\tilde{G}_{n}(\text { two }- \text { port })_{\max }}{\tilde{G}_{1}(\text { one }- \text { port })_{\max }}=1.4
$$

which definitely enlarges the maximum read distance of $20 \%$.

The measured analog identifier associated to chip 1 is shown in Fig. 6. As in the single chip case, the analog identifier is rather independent on the angle except for those frequencies where the realized gain is small, as in the case of observation angles $P_{1}(f=880 \mathrm{MHz})$ or $P_{5}(f=910 \mathrm{MHz})$. In these conditions the reader is processing a low-intensity backscattered signal and the computation in (2) is hence affected by poor accuracy. Nevertheless the measured analog identifier appears considerably 


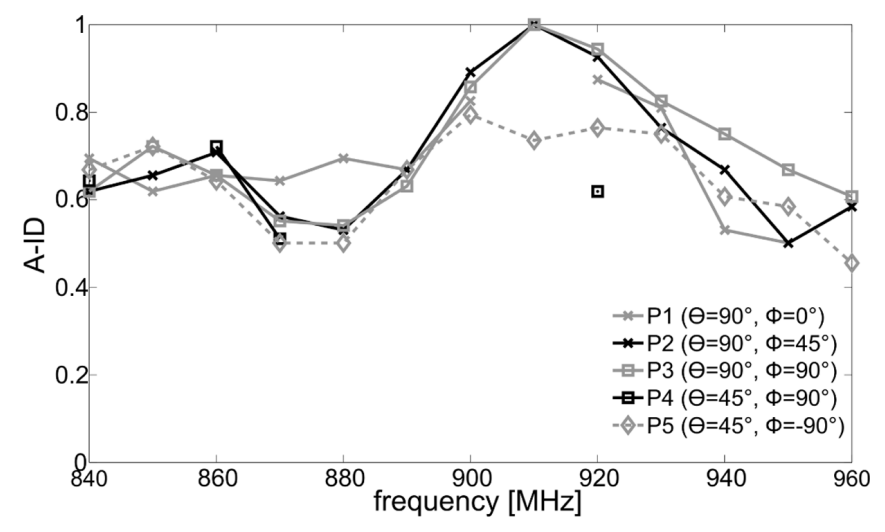

a)

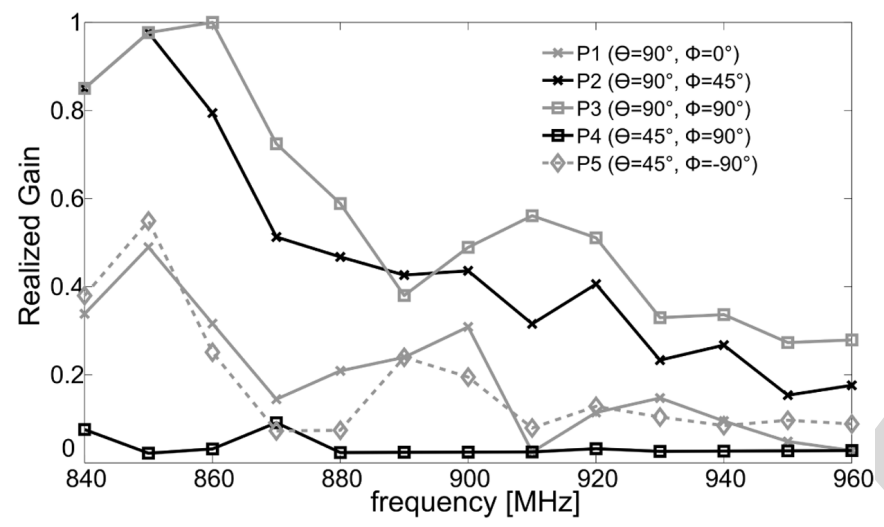

b)

Fig. 6. Two-tags grid: normalized a) A-Id and b) realized gain at some observation angles, $\left(\tilde{G}_{n}\right)_{\max }=2.34$.

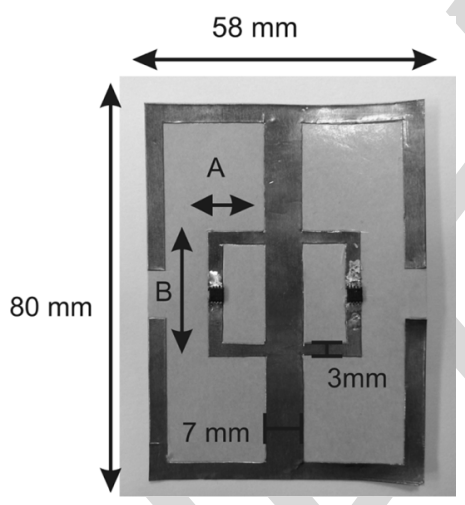

Fig. 7. Two-chip tag: prototype and size from [20].

much more stable than the measurement of the realized gain shown in Fig. 5.

\section{B. Integrated Dual-Chips Tag}

The two-port tag already presented by the authors in [20] to achieve a temperature sensor is here considered to discuss the analog identifier's invariance. The tag (Fig. 7) consists of a partly folded dipole provided with two equal-size T-match circuits to control the port impedance.

The size $(A=12 \mathrm{~mm}, B=25 \mathrm{~mm})$ of the double T-Match were designed to maximize the active power transfer coefficients $\tau_{n}=4 R_{C} R_{S} /\left|Z_{S}+Z_{C}+Z_{M}\right|^{2}$ of the two-ports grid

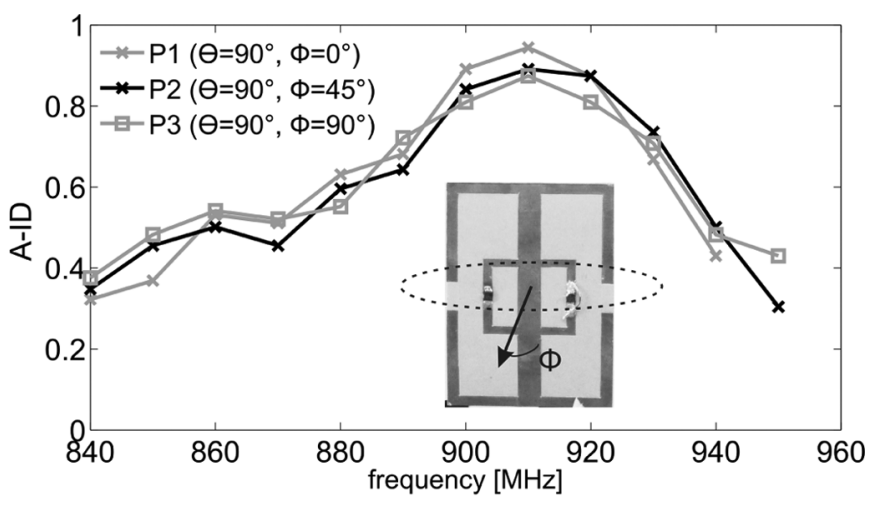

a)

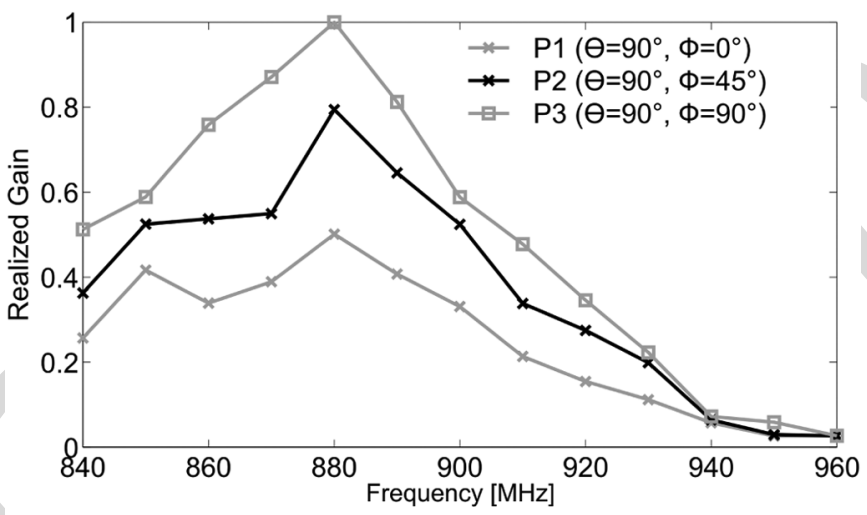

b)

Fig. 8. Two-chips Grid: normalized A-Id and realized gain of one of the ports at some observation angles. Normalization factor for the gain: $\left(\tilde{G}_{n}\right)_{\max }=0.7$.

in the broadside direction having assumed the same NXP microchip as before. An FDTD model of the antenna predicted self and mutual impedance at $870 \mathrm{MHz}$ of $Z_{S}=7.2+j 132$ and $Z_{M}=-5+j 6.25 \Omega$, respectively. Fig. 8 shows the measured analog identifiers of port 1 and the corresponding realized gain at three different observation angles over the horizontal $\left(\theta=90^{\circ}\right)$ plane. The A-Id is even more independent on the observation angle than the previous example, due to the higher values of the realized gains so that the tag will backscatter fields of similar strength, resulting in a more accurate processing of the received power.

\section{Sensing Tags}

The fingerprint-extraction procedure is here applied to the sensing of things and in particular to the detection of the physical (time-varying) state of the tagged object.

For instance, in logistics it is sometimes required to monitor the status of containers (bottles, packages, bags) with the purpose to detect variation of their solid, powder or liquid content. The self-sensing natural capabilities of RFID tags may be exploited to handle these events (see [2] for a complete introduction to RFID self-sensing). The example given here considers the sensing of sugar-powder level inside a plastic container. This is a very challenging problem for RFIDs due to the low-relative permittivity $\left(\epsilon_{r}=2.76, \sigma_{s}=2.44 \cdot 10^{-2} \mathrm{~S} / \mathrm{m}\right)$, which is very similar to that of the air $\left(\epsilon_{r}=1\right)$. The variation of filling level 


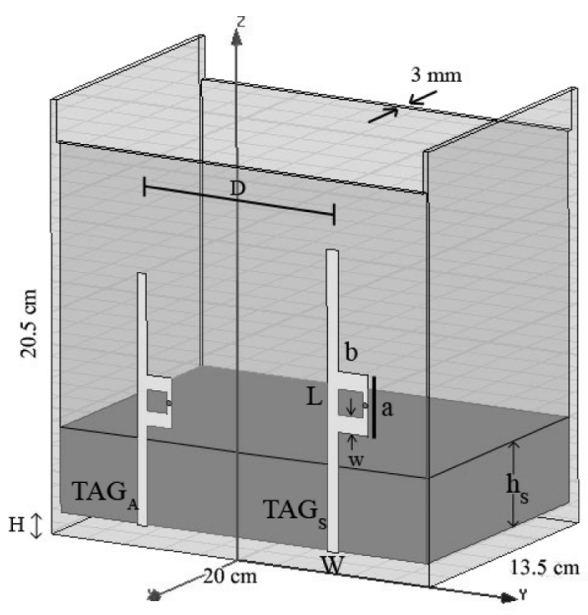

Fig. 9. Two-port grid for sensing the sugar level within a perspex box. Geometrical size (in [mm]): $H=12, D=95 ; T A G_{S} L=142.8, W=6$, $a=28.8, b=15.6, w=8.4 ; T A G_{A} L=119, W=5, a=24, b=13$ $w=7$.

is hence expected to produce only a slight modification of the tag's electromagnetic parameters, and in turn of the sensed data.

The perspex box $\left(\epsilon_{p}=2.7, \sigma_{p} \sim 0\right)$ used for the sugarpowder experiment is shown in Fig. 9. The sensing RFID Grid is now composed by two $\mathrm{T}$-match dipoles of different size attached at a close distance onto a same face of the box. The two tags, denoted as air-tag $\left(T A G_{A}\right)$ and sugar-tag $\left(T A G_{S}\right)$, have been optimized for the best conjugate matching to the NXP microchip when the box is empty $\left(\mathrm{TA}_{A}\right)$ and filled with $\operatorname{sugar}\left(T A G_{S}\right)$, respectively.

The various measurements of the analog identifiers are executed by gradually filling the box with sugar, starting from $h_{S}=0$ up to $h_{S}=14 \mathrm{~cm}$, i.e., roughly corresponding to the height of the longest dipole $T A G_{S}$. The reader and the tags are aligned to have maximum polarization efficiency.

The diagrams in Fig. 10 give the theoretical (computer simulated) and experimental analog identifier for both the tags for three different mutual orientations. The analog identifier of $T A G_{S}$ is rather insensitive (Fig. 10(a)) to the variation of the target, and could be used as a control information, e.g., it could provide the digital "label" of the object. The Analog identifier related to $T A G_{A}$ (Fig. 10(b)) is instead able to sense the sugar variation when the filling level exceeds half the tag size and spans an overall dynamics of about $50 \%$, up to saturation. The collected data are very little dependent on the mutual orientation between the reader and the container, even for rotation of $180^{\circ}$ e.g., when the two antennas are completely shadowed with respect to the reader by the sugar itself.

\section{Two Dimensional $3 \times 3$ GRID}

It is here finally considered a 3 by 3 grid (Fig. 11) made of the commercial tags already used in Section III.

The analog identifier of the central (label 22) and the edge (label 11) elements are measured by the same procedure as before. The invariance of the so obtained fingerprints (Fig. 12) respect to the reader-grid orientation is less sharp than in the previous examples, but nevertheless A-Id is still much more insensitive to rotations than the measurement of the realized gain

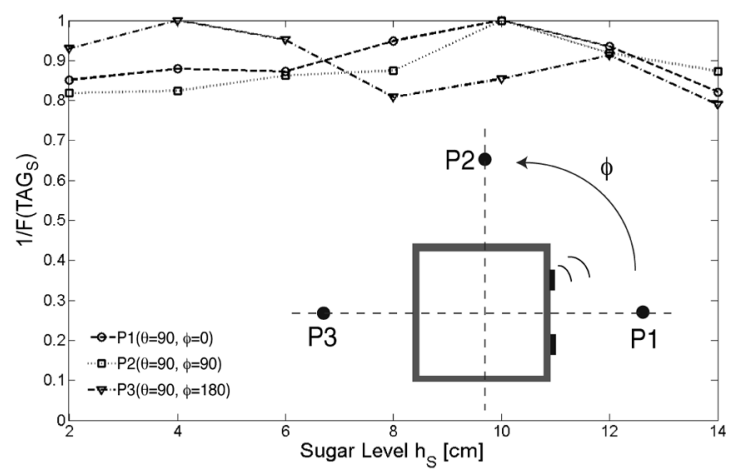

a)

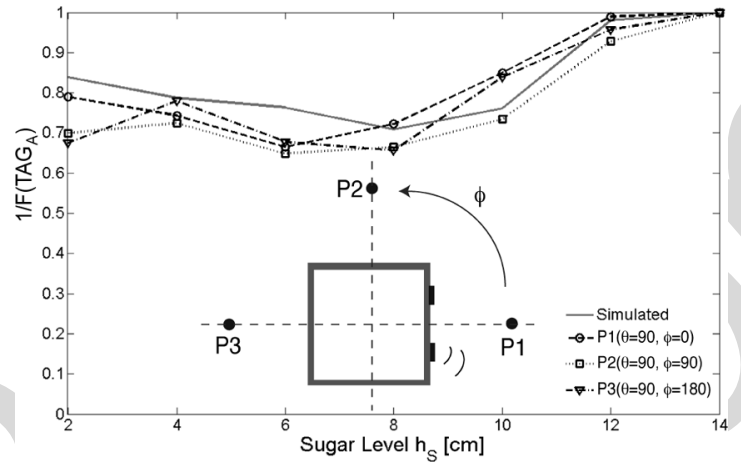

b)

Fig. 10. Two-port grid for Sugar sensing: (Normalized) analog identifiers $F_{n}^{-1}$ for: a) $T A G_{S}$ and b) $T A G_{A}$. The data acquisition has been repeated for different orientation, on the horizontal plane, between reader and tagged box so that the case of $180^{\circ}$ rotation means that the grid is completely shadowed to reader by the box.

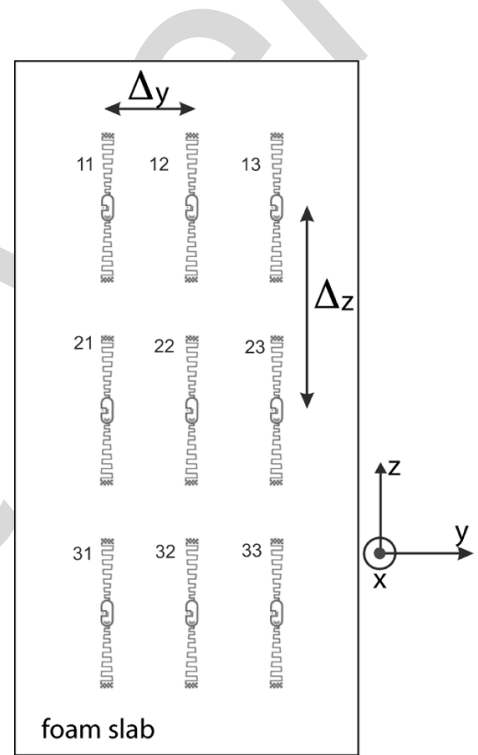

Fig. 11. $3 \times 3$ RFID Grid: the tags are attached over a foam slab. Grid steps: $\Delta_{y}=9 \mathrm{~cm}, \Delta_{z}=14 \mathrm{~cm}$.

(Fig. 13), especially when edge elements are observed. Moreover it is interesting to note that the fingerprints of the two tags are rather similar, unlike the corresponding measured gains.

\section{A. Variable Environment}

The presence of any object close to the reader-tag system can in principle modify the tag responses (turn-on power and 


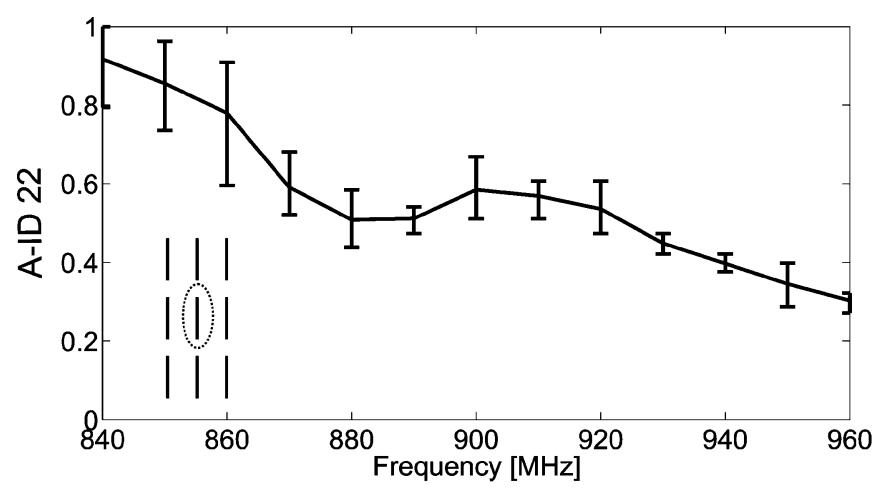

a)

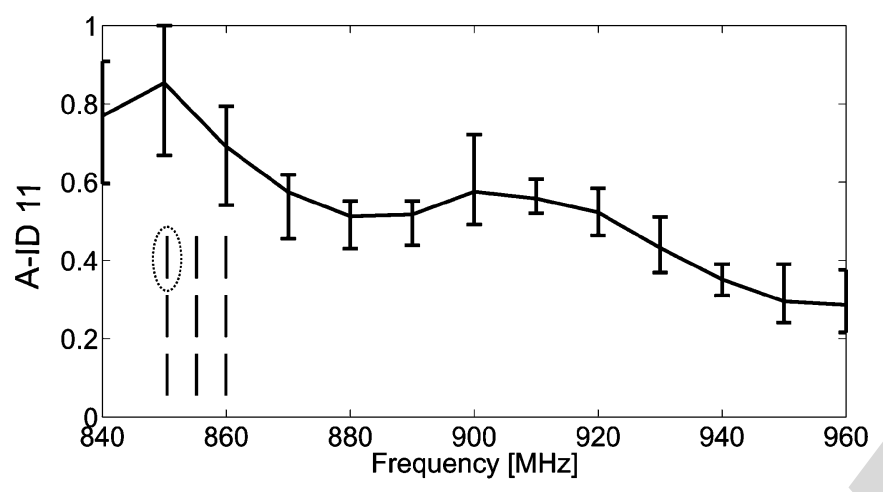

b)

Fig. 12. $3 \times 3$ RFID Grid: measured A-Id of a) central and b) edge elements. The grid plane is $80 \mathrm{~cm}$ far from the reader. The vertical segments give the residual angular variation of A-Id at each frequency sample as the observation angles are randomly chosen in the range $(\theta=90,0<\phi<2 \pi)$. The continuous line gives the angle-average fingerprint.

backscattered power) due to the electromagnetic scattering that it produces. However, since its disturbing effect applies on both the forward and backward links of the RFID communication in the same manner, it can be in principle removed by the calculation of the analog identifier which involves the processing of the turn-on and the backscattered powers.

Assuming that the scatterer is distant enough from the grid so that its input parameters are not significantly modified, the presence of the scatterer will therefore affect only the radiation pattern of the reader and of the grid. However, since the definition of A-Id drops out the reader's gain, the obtained $\left\{F_{n}\right\}$ are expected to be "transparent" to the presence of the scatterer which, in this sense, becomes "invisible" for the grid.

To verify this property, a vertical metallic cylinder, $180 \mathrm{~cm}$ high and $7 \mathrm{~cm}$ in diameter, has been placed in the region between the reader and the grid under test (Fig. 14), close to Line Of Sight condition. It was actually at a distance of $20 \mathrm{~cm}$ from the reader antenna and moved $11 \mathrm{~cm}$ sideways from the line of sight, first on the left and later on the right. The grid is measured in the fixed direction $\left(\phi=0^{\circ}, \theta=90^{\circ}\right)$.

The A-Id of the central tag is shown in Fig. 15(a) and compared to the realized gain of the same configuration $15 \mathrm{~b}$ ). This experiment is considered very challenging since the big metallic object is expected to produce a significant scattering and to seriously affect the measurement of the realized gain of grid's elements, as clearly visible in Fig. 15(b). Instead, the analog identi-

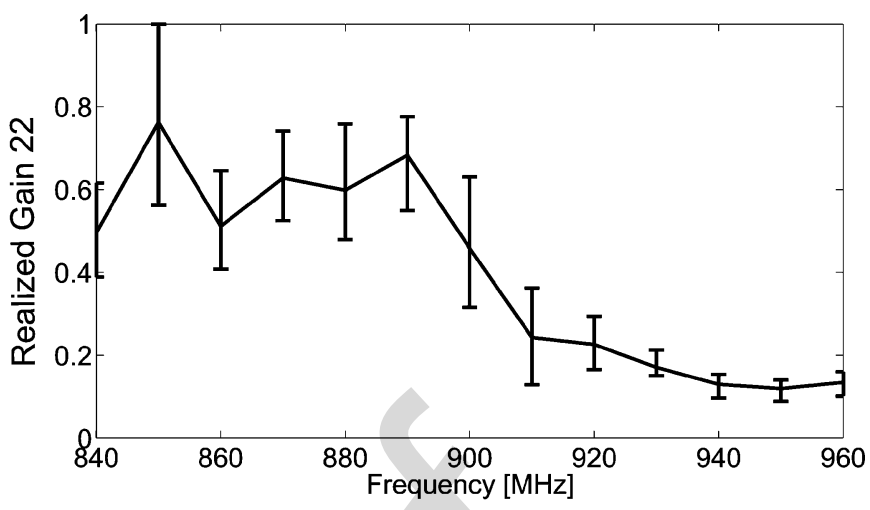

a)

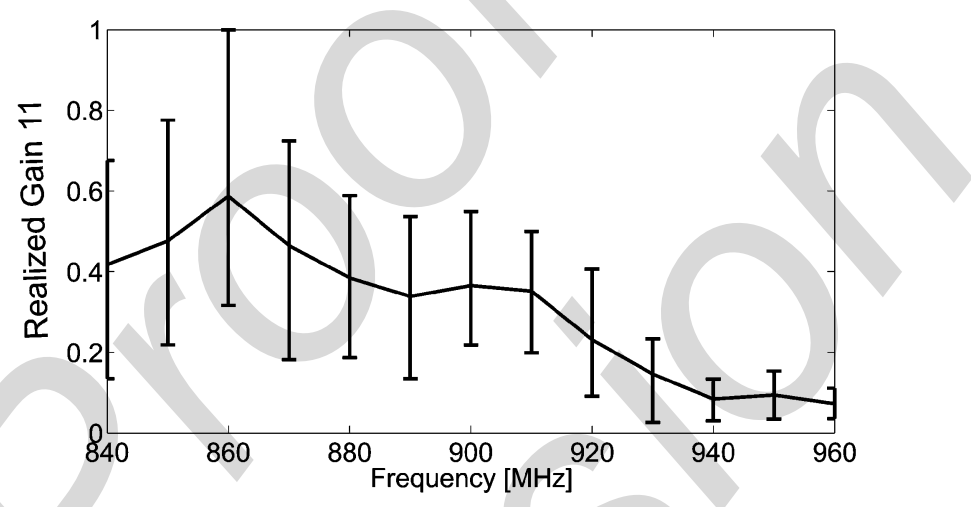

b)

Fig. 13. $3 \times 3$ RFID Grid: normalized measured realized gain of a) central element (maximum gain used for the normalization: $\tilde{G}_{22, \max }=0.29$ ) and b) edge element $\left(\tilde{G}_{11, \max }=0.53\right)$. Diagram's conventions as in Fig. 12.

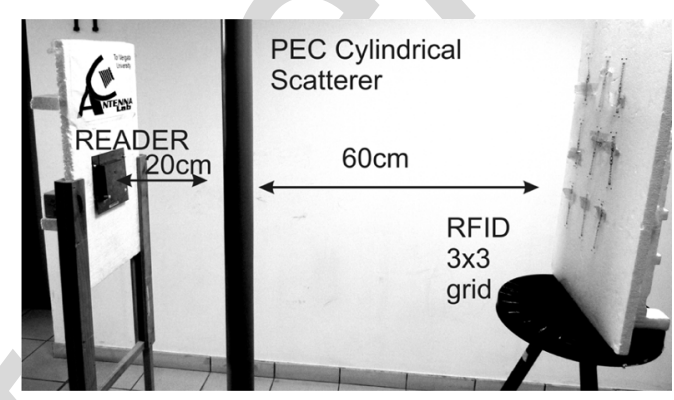

Fig. 14. $3 \times 3$ RFID Grid in variable environment: Measurement setup, comprising a RFID reader (left), the $3 \times 3$ RFID grid (right) and the metallic cylindrical scatterer interposed between the reader and the grid.

fier in Fig. 15(a) revealed also in this case to be really insensitive to the presence and to the position of the scatterer, and by extrapolation, to the motion of nearby objects or people provided that the characteristic times of such motion are large compared with the RFID interrogation rate. However, it is worth mentioning that the cancellation of the scatterer is not an exclusive property of the GRID arrangement, but it can be achieved also with just a standalone tag, as shown in the experiment in Fig. 16 where a single element of the array is placed close to the pipe scatterer as in the previous configuration.

\section{CONCLUSIONS}

The presented experimentations corroborate the theoretical analysis of RFID grids. The formulas for the active realized 


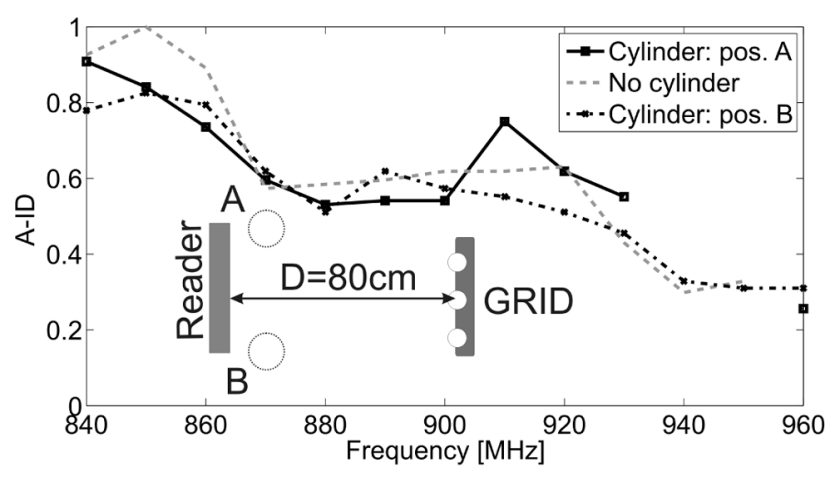

a)

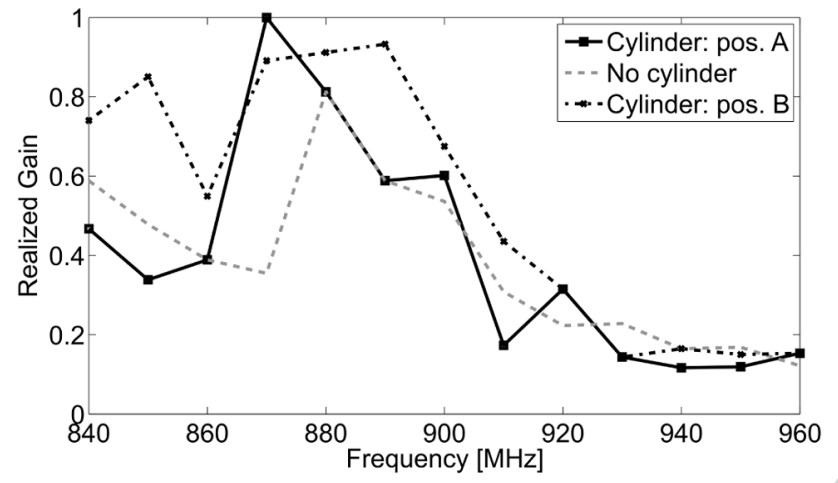

b)

Fig. 15. $3 \times 3$ RFID Grid: a cylindrical scatterer moved close to the reader/tag system into two different positions A and B: a) analog identifier of the central (22) tag collected for mutual reader-tag orientation $\phi=0^{\circ}, \theta=90^{\circ}$ and b) normalized realized gain.

gain and the impedance matching can be used together with any standard optimization design procedure. The inter-port coupling, which is generally considered as a negative effect of tags' proximity, can be instead judiciously handled to enlarge the read range of each microchip, or for the same distance, to reduce the required power.

The tag's fingerprint obtained by the calculation of the analog identifier seems to be valuable in both single and multi-chip configurations. This quantity demonstrated to be rather insensitive to the many uncertain conditions of typical RFID interrogations in a real world, e.g., to its mutual position with respect to the grid, as well as to the variation of the environment itself. However, since the definition of the fingerprint is based on the accurate measurement of the turn-on power and of the backscattered power, the true reliability of the analog identifier, for instance during sensing or security assessment, can not leave apart the quality of the reader's receiver. It is reasonable to assume that even more robust results could be achieved by using the value of the backscattered power as a quality factor of the measurements.

The cost of grid-based devices is expected to be higher than that of standard tags used as simple wireless replacement of barcodes. However multi-chips tags will offer augmented features making them closer to sensors than to radiofrequency labels.

Application to sensing appears indeed promising and in future experiments a multi-chip sensing grid could be empowered by a plurality of chemical receptors to achieve multi-variant

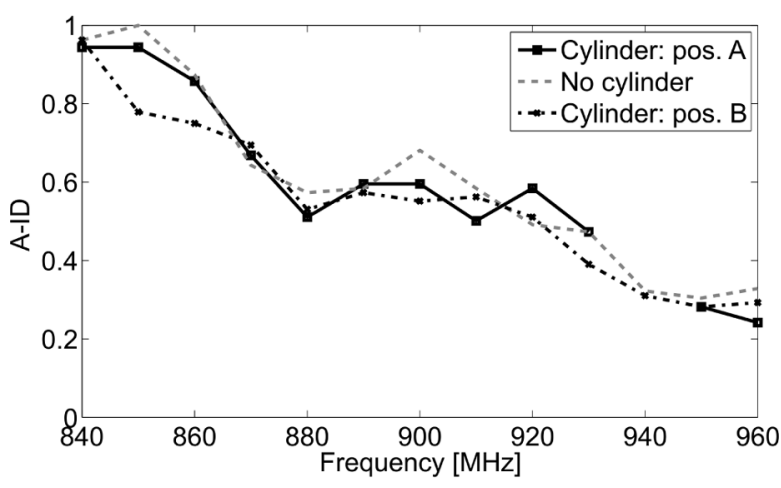

a)

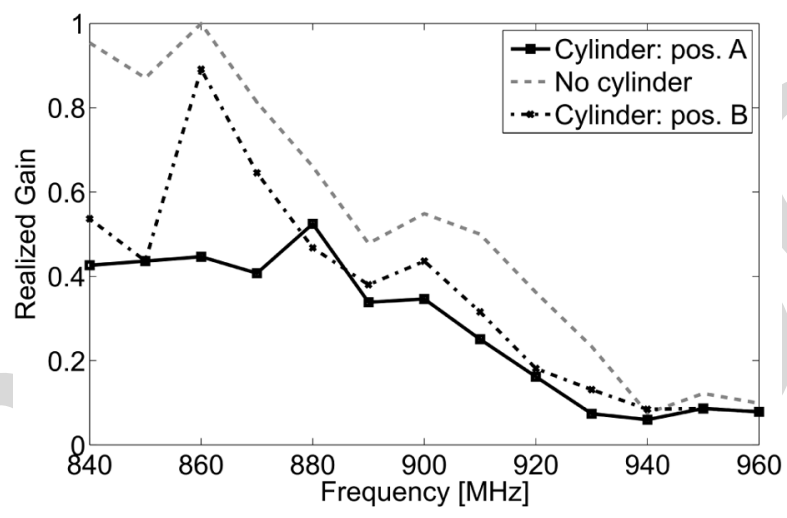

b)

Fig. 16. Single tag in presence of a variable environment in the same conditions as in the $3 \times 3$ array case. a) analog identifier b) normalized realized gain.

TABLE I

Sizes of THE Designed Single TAg AND Coupled TAgS

\begin{tabular}{c|c|c} 
& Coupled Tags $(\mathrm{mm})$ & Single Tag $(\mathrm{mm})$ \\
\hline$L_{t o t}$ & 155 & the same \\
\hline$W$ & 7 & the same \\
\hline$A$ & 10 & 16 \\
\hline$B$ & 12 & 16 \\
\hline$\triangle$ & 80 & -
\end{tabular}

low-cost remote sensors. The fingerprint concept will give a reasonable confidence that a change in the grid's responses in successive interrogations will be related to a change of the physical parameters under test, rather than to a change of the mutual reader-tag position. So, there will be many opportunities to develop new devices but also there will be the need of ad-hoc data retrieval algorithms to master specific applications.

\section{REFERENCES}

[1] G. Marrocco, "RFID grids: Part I-Electromagnetic theory," IEEE Trans. Antennas Propag..

[2] G. Marrocco, L. Mattioni, and C. Calabrese, "Multi-port sensor RFIDs for wireless passive sensing-Basic theory and early simulations," IEEE Trans. Antennas Propag., vol. 56, no. N.8, pp. 2691-2702, Aug. 2008.

[3] M. Philipose, J. R. Smith, B. Jiang, A. Mamishev, S. Roy, and K. Sundara-Rajan, "Battery-free wireless identification and sensing," IEEE Perv. Comput., vol. 4, no. N.1, pp. 37-45, Jan.-Mar. 2005.

[4] P. Nikitin, K. V. S. Rao, and S. Lam, "RFID Tags With Enhanced Range and Bandwidth Obtained by Spatial Antenna Diversity," U.S. Patent no. US2009/ 0219158, Sep. 2008. 
[5] J. W. Wallace and M. A. Jensen, "Mutual coupling in MIMO wireless systems: A rigorous network theory analysis," IEEE Trans. Wireless Commun., vol. 3, no. N.4, pp. 1317-1325, Jul. 2004.

[6] D. Preuveneers and Y. Berbers, "Internet of things: A context- awareness perspective," in The Internet of Things: From RFID to the NextGeneration Pervasive Networked Systems, L. Yan, Y. Zhang, L. T. Yang, and H. Ning, Eds. London: Auerbach Publications, 2008.

[7] L. Ukkonen and L. Sydanheimo, "Threshold power-based radiation pattern measurement of passive UHF RFID tags," in Proc. PIERS, Cambridge, 2010, pp. 87-90.

[8] P. V. Nikitin and K. V. S. Rao, "Theory and measurement of backscattering from RFID tags," IEEE Antennas Propag. Mag., vol. 48, no. N. 6, pp. 5459-5462, Dec. 2006.

[9] S. Skali, C. Chantepy, and S. Tedjini, "On the measurement of the delta radar cross section ( $\triangle \mathrm{RCS})$ for UHF tags," in Proc. IEEE Int. Conf. on RFID 2009, pp. 346-351.

[10] S. L. Chen, K.-H. Lin, and R. Mittra, "A measurement technique for verifying the match condition of assembled RFID tags," IEEE Trans. Instrum. Meas., vol. 59, no. 8, Aug. 2010.

[11] J. P. Daniel, "Mutual coupling between antennas for emission and reception- application to passive and active dipoles," IEEE Antennas Propag. Mag., vol. 22, no. 2, pp. 347-349, 1974.

[12] F. Lu, X. Chen, and T. T. Ye, "Performance analysis of stacked RFID tags," in Proc. IEEE Int. Conf. on RFID, 2009, pp. 330-337.

[13] K. Lee and T. Chu, "Mutual coupling mechanisms within arrays of nonlinear antennas," IEEE Trans. Electromagn. Compat., vol. 47, no. 4, pp. 963-970, 2005.

[14] X. Chen, F. Lu, and T. T. Ye, "The weak spots in stacked UHF RFID tags in NFC applications," in Proc. IEEE Int. Conf. on RFID, 2010, pp. $181-186$.

[15] V. Rizzoli, A. Costanzo, M. Rubini, and D. Masotti, "Rigorous investigation of interactions between passive RFID tags by means of nonlinear/electromagnetic co-simulation," in Proc. Eur. Microwave Conf., 2006, pp. 722-725.

[16] Y. Tanaka, Y. Umeda, O. Takyu, M. Nakayama, and K. Kodama, "Change of read range for UHF passive RFID tags in close proximity," in Proc. IEEE Int. Conf. on RFID, 2009, pp. 338-345.

[17] J. D. Griffin and G. D. Durgin, "Complete link budgets for backscatterradio and RFID systems," IEEE Antennas Propag. Mag., vol. 51, no. N.2, pp. 11-25, 2009.

[18] J. Virtanen, L. Ukkonen, T. Bjorninen, and L. Sydanheimo, "Printed humidity sensor for UHF RFID systems," IEEE Sensors Applicat., pp. 269-272, 2010

[19] G. Marrocco, "The art of UHF RFID antenna design: Impedance matching and size-reduction techniques," IEEE Antennas Propag. Mag., vol. 50, no. N.1, pp. 66-79, Feb. 2008.

[20] S. Caizzone, C. Occhiuzzi, and G. Marrocco, "Multi-chip RFID antenna integrating shape-memory alloys for detection of thermal thresholds," IEEE Trans. Antennas Propag., to be published.

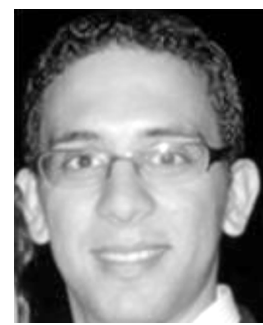

tennas.

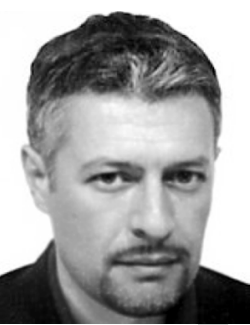

Gaetano Marrocco was born in Teramo, Italy, on August 29, 1969. He received the Laurea degree in electronic engineering (Laurea cum laude and Academic Honour) and the Ph.D. degree in applied electromagnetics from the University of L'Aquila, Italy, in 1994 and 1998, respectively.

Since 1997, he has been a Researcher at the University of Rome "TorVergata," Rome, Italy, where he currently teaches Antenna Design and Medical Radio-Systems, manages the Antenna Lab and is Advisor in the Geo-Information Ph.D. program. In October 2010, he achieved the level of Associate Professor of electromagnetic. In summer 1994, he was at the University of Illinois at Urbana-Champain as a Postgraduate student. In autumn 1999, he was a Visiting Researcher at the Imperial College in London, U.K. In 2008, he joined the Ph.D. program of the University of Grenoble (FR). His research is mainly directed to the modeling and design of broad band and ultrawideband (UWB) antennas and arrays as well as of sensor-oriented miniaturized antennas for biomedicine, aeronautics and radiofrequency identification (RFID). He has been involved in several Space, Avionic, Naval and Vehicular programs of the European Space Agency, NATO, Italian Space Agency, and the Italian Navy about the analysis and the design of non conventional antennas and systems. He holds eight patents on broadband naval antennas and structural arrays, and on sensor RFID systems.

Prof. Marrocco currently serves as an Associate Editor of the IEEE Antennas and Wireless Propagation Letters, he is a reviewer for the IEEE TRANSACTION on ANTENNAS AND PROPAGATION, IEEE PROCEEDINGS, IEEE TRANSACTIONS ON MICROWAVE THEORY AND TECHNIQUES, and is a member of Technical Program Committee of several International Conferences. In 2008, he was the General Chairman of the first Italian multidisciplinary scientific workshop on RFID: RFIDays-2008: Emerging Technology for Radiofrequency Identification. He was the Co-Chair of the RFIDays-2010 International Workshop in Finland and Chairman of the Local Committee of the V European Conference on Antennas and Propagation. 